\title{
The Jurassic-Early Cretaceous basalt-chert association in the ophiolites of the Ankara Mélange, east of Ankara, Turkey: age and geochemistry
}

\author{
VALERIO BORTOLOTTI*, MARCO CHIARI $+\dagger$, M. CEMAL GÖNCÜOGLU§, \\ GIANFRANCO PRINCIPI*, EMILIO SACCANIף, U. KAGAN TEKIN\| \\ \& RENZO TASSINARI \\ * Dipartimento di Scienze della Terra, Università degli Studi di Firenze, Via G. La Pira 4, 50121 Firenze, Italy \\ tCNR, Istituto di Geoscienze e Georisorse, Via G. La Pira 4, 50121 Firenze, Italy \\ $\S$ Geological Engineering Department, Middle East Technical University, 06531, Ankara, Turkey \\ -Dipartimento di Fisica e Scienze della Terra, Università degli Studi di Ferrara, Via Saragat 1, 44122 Ferrara, Italy \\ ||Geological Engineering Department, Hacettepe University, 06532, Beytepe, Ankara, Turkey
}

(Received 30 October 2016; accepted 10 April 2017; first published online 6 June 2017)

\begin{abstract}
This study is focused on slide blocks including oceanic lavas associated with pelagic sediments within the eastern part of the Ankara Mélange. A detailed petrological characterization of the volcanic rocks and a detailed biochronological investigation of the associated radiolarian cherts in eight sections (east of Ankara) was carried out. The volcanic rocks are largely represented by basalts and minor ferrobasalts and trachytes. They show different geochemical affinities and overlapping ages including: (a) Late Jurassic - Early Cretaceous garnet-influenced MORB (middle late Oxfordian to late Kimmeridgian-early Tithonian and early-early late Tithonian; late Valanginianearly Barremian); (b) Early Cretaceous enriched-MORB (middle late Barremian-early early Aptian; Valanginian to middle Aptian-early Albian); (c) Middle Jurassic plume-type MORB (early-middle Bajocian to late Bathonian-early Callovian); (d) Late Jurassic - Early Cretaceous alkaline basalts (middle-late Oxfordian to late Kimmeridgian-early Tithonian; late Valanginian to late Hauterivian). All rock types show a clear garnet signature, as testified to by their high MREE/HREE (middle rare earth element/heavy rare earth element) ratios. The coexistence of chemically different rock types from Middle Jurassic to Early Cretaceous times suggests that they were formed in a mid-ocean ridge setting from partial melting of a highly heterogeneous mantle characterized by the extensive occurrence of OIB-metasomatized portions, which were likely inherited from Triassic mantle plume activity associated with the continental rift and opening of the Neotethys branch.
\end{abstract}

Keywords: ophiolites, geochemistry, radiolarian biostratigraphy, Jurassic, Early Cretaceous, Ankara Mélange, Turkey

\section{Introduction}

Despite numerous studies since the 1960s in Turkey, the locations, ages and geological evolution of the branches of the Eastern Mesozoic Tethys Ocean or Eastern Neotethys are disputed (see Sengör \& Y1lmaz, 1981; Dercourt et al. 1986; Robertson et al. 1996; Göncüoglu, Dirik \& Kozlu, 1997; Dilek et al. 1999; Göncüoglu et al. 2000; Stampfli \& Borel, 2002; Bortolotti \& Principi, 2005; Göncüoglu, Yaliniz \& Tekin, 2006; Schmid et al. 2008; Moix et al. 2008; Göncüoglu, Sayit \& Tekin, 2010). This is mainly owing to two deficiencies. One of them is the fact that next to definite ophiolitic suture belts separating continental blocks with distinct geological histories there are also suture-like alignments or pseudo-belts of allochthonous ophiolitic bodies. The second shortcoming regards the method of ophiolite research yet realized, as it was rarely based upon a multidisciplinary

$\dagger$ Author for correspondence: marco.chiari@unifi.it approach. Several detailed petrological studies on different units from different ophiolitic bodies lack age data and vice versa. A considerable number of studies were concentrated on the larger and more or less complete ophiolitic bodies and neglected the remarkable amount of basalt-radiolarian chert associations within the mélange complexes. To overcome these problems we applied a multidisciplinary approach combining petrology and biostratigraphy on crustal remnants of the Neotethys incorporated into the mélange complexes of the Izmir-Ankara-Erzincan Suture Belt (IAESB).

As previously referred to, the location, age and geological evolution of the branch(es) of the Eastern Neotethys are disputed. In fact, most authors favour the hypothesis (presented herein) that implies the existence of two parallel and contemporaneous oceans, namely, the Izmir-Ankara Ocean, to the south, and the Intra-Pontide Ocean, to the north (see Göncüoglu et al. 2012 for an exhaustive review). In contrast, other authors favour the hypothesis that implies 


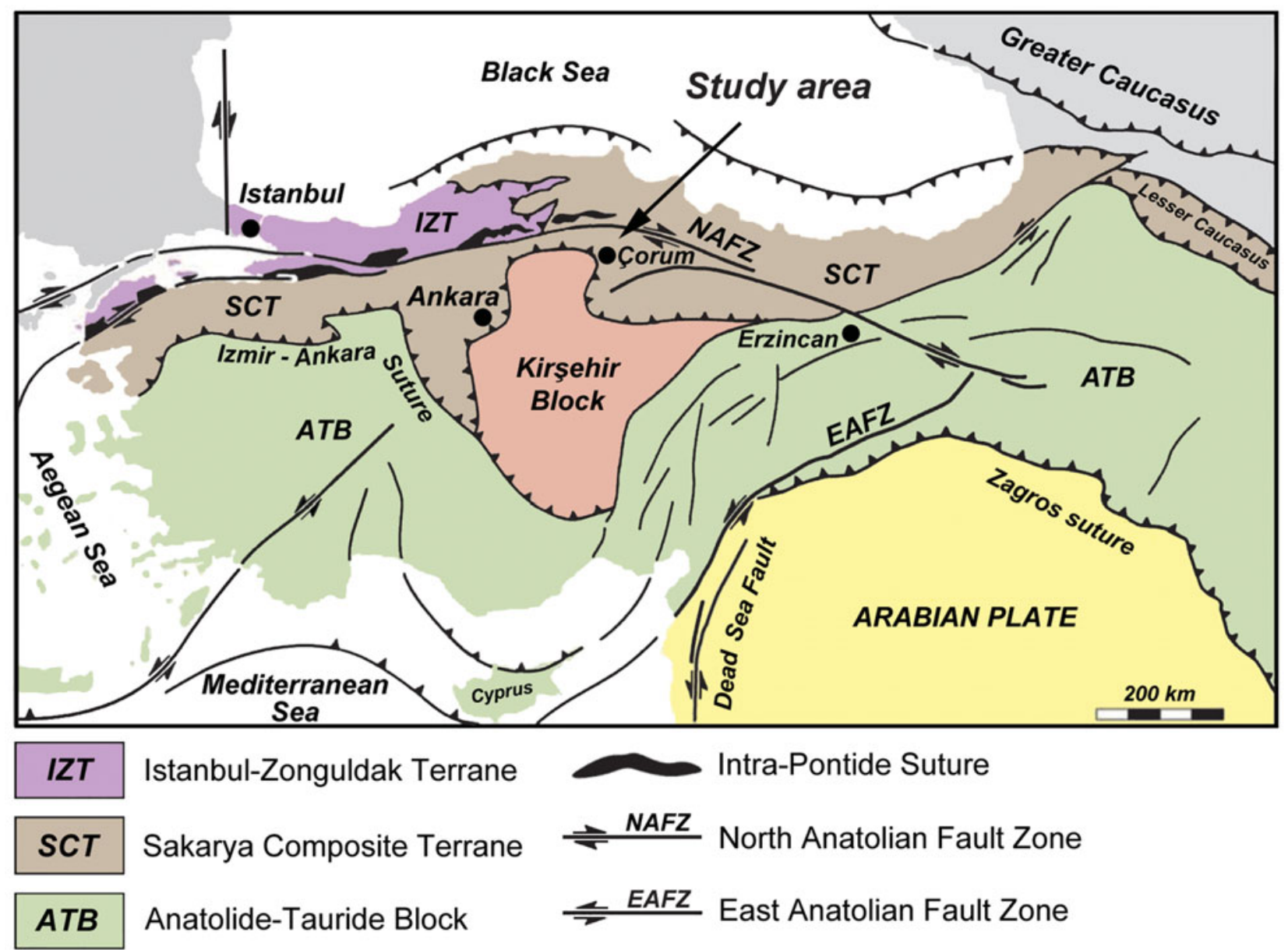

Figure 1. (Colour online) The main tectonic zones of Turkey (modified after Sengör \& Y1lmaz, 1981; Göncüoglu et al. 2012, modified).

the existence of a single oceanic basin represented by the Intra-Pontide Ocean (e.g. Bortolotti \& Principi, 2005). In this case the Intra-Pontide Suture (IPS, see below) would be the eastward continuation of the Vardar Ocean suture, whereas the IAESB would represent fragments of the oceanic lithosphere pushed southwards onto the continent by orogenic movements (as happened more westwards, in the Hellenides) (see Saccani et al. 2008a; Bortolotti et al. 2013b).

The IAESB separates two completely different units of continental crust with different origins: (a) the Tauride-Anatolide terrane representing the rifted northern margin of NW Gondwana, in the south, and (b) the Sakarya Composite Terrane comprising amalgamated oceanic and continental bodies of Variscan and Cimmerian origin and their alpine platform, in the north. To the north of the Sakarya Composite Terrane another suture, the IPS, represents the boundary towards the Eurasian Istanbul-Zonguldak terrane (Fig. 1).

The IAESB represents remnants of the VardarIzmir-Ankara-Erzincan-Lesser Caucasus Ocean in Turkey. This ocean is considered the main northern branch of the Neotethys, whereas the Intra-Pontide oceanic basin to the north of it is a matter of de- bate (see above and, for a brief discussion, Göncüoglu et al. 2012 and Tekin et al. 2012). In NW Turkey, the IPS is clearly delineated by the presence of ophiolitic melanges between the Sakarya and the Istanbul-Zonguldak continental plates (e.g. Robertson \& Ustaömer, 2004; Göncüoglu et al. 2008; Akbayram, Okay \& Satir, 2012). By the wedging out of the Sakarya Composite Terrane in northern Central Anatolia, however, the IPS belt juxtaposes with the IAESB along splays of the North Anatolian Shear Zone (e.g. Ellero et al. 2015a) and the neotectonic strike-slip system generated owing to the Tertiary indentation and anticlockwise rotation of the Kirşehir Block (e.g. Cemen et al. 1993; Kaymakci et al. 2003). Towards the east and in NE Anatolia, the IAESB is again in its accustomed position between the Anatolides and the units of the Sakarya Composite Terrane (Topuz et al. 2013a,b; Parlak et al. 2013; Robertson et al. 2013). The vanishing of the IPS belt in the Central Pontides by the North Anatolian Shear Zone during the neotectonic period led some authors to completely ignore the IPS and hence the presence of an oceanic basin (e.g. Elmas \& Yiğitbaş, 2001, 2005).

The IAESB is composed of a number S-verging tectonic slices or giant slide blocks representing (1) 
incomplete sequences of various portions of the IzmirAnkara-Erzincan oceanic lithosphere (e.g. Floyd et al. 2000; Göncüoglu et al. 2000; Göncüoglu, 2011); (2) an accretionary prism, known as the 'Ankara Mélange' (Bailey \& McCallien, 1953) including blocks derived from different parts of the oceanic basin; (3) imbricated slices of successions formed in a foredeep basin associated with the emplacement of the oceanic material onto the Tauride-Anatolide passive margin.

This study is focused on slide blocks including oceanic lavas associated with pelagic sediments within the accretionary prism and the foredeep basin successions in the eastern part of the Ankara Mélange (for the preliminary data see Bortolotti et al. 2013a). This paper aims to draw a more comprehensive picture of the different basaltic rock types erupted in the Neotethys from Middle Jurassic to Early Cretaceous times. To this purpose, a detailed petrological and geochemical characterization of the volcanic rocks and a detailed biochronological investigation of the associated radiolarian cherts will be carried out. Similar multidisciplinary studies were applied to other parts of the IAESB between the Aegean coast and the western part of the Ankara Mélange (e.g. Bragin \& Tekin, 1996; Yaliniz, Göncüoglu \& Floyd, 2000; Göncüoglu, Tekin \& Turhan, 2001; Rojay, Yaliniz \& Altiner, 2001; Tekin, Göncüoglu \& Turhan, 2002; Gökten \& Floyd, 2007; Tekin \& Göncüoglu, 2007, 2009; Göncüoglu et al. 2008; Göncüoglu, Sayit \& Tekin, 2010; Tekin et al. 2012; Moix \& Goričan, 2013; Göncüoglu et al. 2015; Soycan, Erdoğan \& Konak, 2015). These studies reported Middle Triassic to Cretaceous ages for radiolarian cherts stratigraphically associated with basalts showing, in turn, different tectonomagmatic settings of formation, such as mid-ocean ridge, seamount, forearc and back-arc. Our new data from the eastern Ankara Mélange aim to interpret the tectonomagmatic processes and their timing, which will be useful for improving the reconstruction of the geodynamic history of the Neotethys Ocean mainly spanning the Jurassic to Early Cretaceous time interval.

\section{Geological background}

The studied portion of the IAESB was characterized by the pre-Eocene development of a huge accretionary prism (for details see Rojay, 2013), which formed between the Sakarya Composite Terrane to the north and the southerly Kütahya-Bolkardag Belt of the Anatolides, in the western part, and the Central Anatolian Crystalline Complex (CACC), in the eastern part. The first juxtaposition of these oceanic and continental units occurred in latest Cretaceous - Paleocene time. However, compression and related thrusting lasted until Miocene time (Kocyigit et al. 1995).

We sampled some ophiolitic outcrops from the IAESB east of Ankara (Fig. 1), where the concave E$\mathrm{W}$ trend of the belt between Izmir and Ankara makes a sharp turn towards the NNW, caused by the Tertiary indentation (e.g. Cemen et al. 1993; Kaymakci et al. 2003) and anticlockwise rotation of the Kirşehir Block.

The Sakarya Unit marks the active margin of the Izmir-Ankara-Erzincan Ocean, and was thrust over the IAESB both to the NNE of Ankara and to the SE of Çorum (Fig. 2). It consists of a composite terrane comprising a Variscan arc complex and its Permian platform, as well as the Palaeotethyan subduction-accretion prism (the Triassic Karakaya Complex; Göncüoglu et al. 2000; Okay \& Göncüoglu, 2004). It has a Jurassic to ?Upper Cretaceous cover that belonged to a $\mathrm{N}$-facing passive continental margin but was transformed into an active margin by the northward subduction of the Izmir-Ankara-Erzincan oceanic lithosphere.

The Karakaya Complex NNW of Ankara comprises low-grade metamorphic greywackes associated with Permian and Carboniferous limestone blocks and ocean-island-type volcanic rocks with Carnian radiolarian cherts (e.g. Sayit, Tekin \& Göncüoglu, 2011). Unconformably overlying them there is a Mesozoic cover that comprises, from bottom to top, upper Lower Jurassic - Middle Jurassic neritic limestones, Upper Jurassic - Lower Cretaceous (e.g. Altiner et al. 1991) pelagic limestones and Upper Cretaceous turbidites. The oldest common overstep sequence on the IAESB (and the Sakarya Composite Terrane) comprises Upper Paleocene lagoonal sediments, which occur as discontinuous outcrops within the thrust zone between these two units (Göncüoglu et al. 2000).

The northern-central part of the sampled area has been recently evaluated as the North Anatolian Shear Zone (Ellero et al. 2015a,b). The North Anatolian Shear Zone corresponds to a complex deformation zone where the strain is partitioned in a system of faults, folds and thrusts leading to high-angle faults bounding E-W-elongated blocks and pull-apart basins. The zone is more than $100 \mathrm{~km}$ wide and comprises several kilometres long, lens-shaped tectonic inlayers belonging to the mélanges of the IPS belt (e.g. Göncüoglu et al. 2014), the Sakarya Composite Terrane (mainly the Upper Jurassic - Lower Cretaceous Sogukcam Limestone, e.g. Catanzariti et al. 2013), a Late Cretaceous island arc (Berber, Göncüoglu \& Sayit, 2014) belonging to the IPS belt, a Late Cretaceous continental arc (Ellero et al. 2015b) and the ophiolitic mélanges of the IAESB. The active main strand of the right-lateral North Anatolian Transform Fault is located in the centre of this megashear zone, where lateral displacements ranging from 30 to $120 \mathrm{~km}$ have been proposed in the literature (e.g. Hubert-Ferrari et al. 2002 and references therein).

The metamorphic rocks of the Sakarya Composite Terrane (the 'Tokat Massif', Yilmaz et al. 1997), around Çorum, east of the sampled area (Fig. 2), are tectonically overlying the ophiolitic mélange of the IAESB. In this area, both units are in turn unconformably overlain by Middle Eocene rocks. Unfortunately, the primary relations between the Sakarya and IAESB 


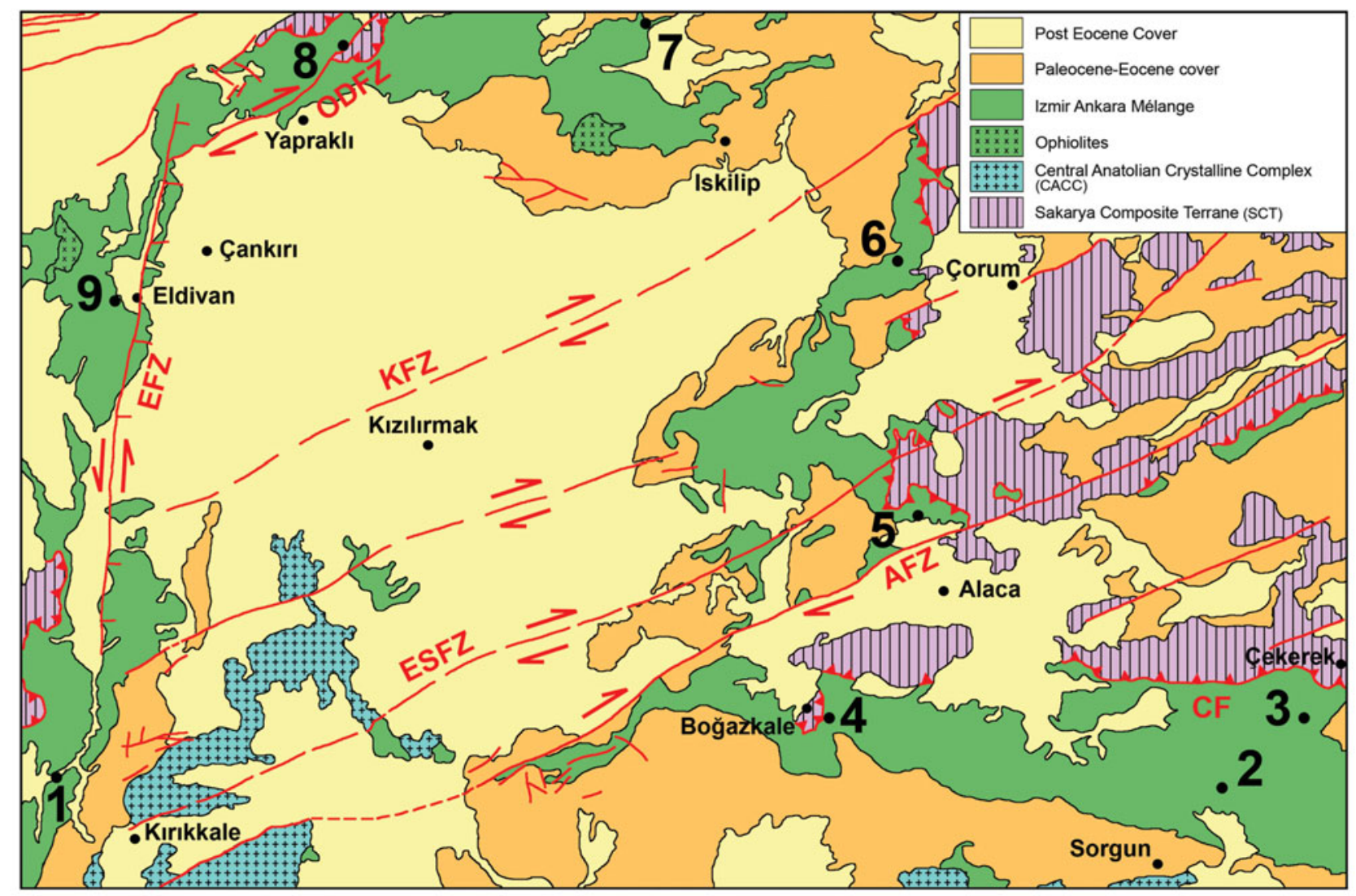

Figure 2. (Colour online) Sketch geological map of the study area, with the location of the sampled sections (after Bortolotti et al. $2013 a$, modified and Yolsal-Çevikbilen et al. 2012). EFZ - Eldivan Fault Zone; ODFZ - Orta-Devrez Fault Zone; KFZ - Kızılırmak Fault Zone; ESFZ - Ezine Pazarı-Sungurlu Fault Zone; AFZ - Alaca Fault Zone; CF - Çekerek Fault.

rocks are obscured by intensive Oligocene strike-slip faulting (Fig. 2).

Structurally underlying the IAESB units, the continental crust that was palinspastically located to the south, and west of Ankara, represents the northern rim of the Tauride-Anatolide Platform. It mainly includes high-pressure-low-temperature metamorphosed tectonic slices (e.g. Okay \& Tüysüz, 1999) whose lithostratigraphic sequences are similar to the Palaeozoic-Mesozoic slope-type successions of the Anatolides (Göncüoglu, 2011). In particular, in the sampled area (Fig. 2), the IAESB units were thrust onto the CACC (Yaliniz, Floyd \& Göncüoglu, 1996; Yaliniz, Göncüoglu \& Özkan-Altiner, 2000), which comprises high-temperature-medium-pressure metamorphic successions similar to the PalaeozoicMesozoic Tauride-Anatolide Platform. In turn, they were overthrust by Turonian supra-subduction ophiolites: the Central Anatolian Ophiolites (Yaliniz, Göncüoglu \& Floyd, 2000), which represent remnants of the Izmir-Ankara-Erzincan Ocean. Late Campanian granitoids intrude both the basement rocks and the overlying ophiolite units (Köksal \& Göncüoglu, 2008), indicating a Late Cretaceous age for the inception of obduction. The oldest overstep sequences in this area are again post-Maastrichtian pre-Lower Eocene (Gülyüz et al. 2013) red conglomerates, testifying to a
Paleocene age for the main juxtaposition of the CACC and IAESB mélanges.

In the central part of the sampled area, the IAESB rocks are covered by the Paleocene-Eocene postorogenic marine clastic-volcaniclastic rocks, carbonates and volcanic rocks of the Çankırı Basin, which, in turn, are partly covered by Neogene sediments (e.g. Göncüoglu, 1992). Within the basin the anticlockwise rotation of the CACC induced the formation of several NE-SW-trending right-lateral faults and, at its western margin, important left-lateral faults (Fig. 2). When restored, the IAESB palinspastically follows also a roughly $\mathrm{E}-\mathrm{W}$ trend and the present distortion of the main tectonic units is re-established.

To attempt a reconstruction of the thickness of the IAESB rocks that compose the subducted and accreted remnants of the Izmir-Ankara-Erzincan oceanic lithosphere, together with island arc and sedimentary rocks of several Late Cretaceous - Paleocene piggy-back basins (e.g. Cater et al. 1991; Erdogan, Akay \& Ugur, 1996), geophysical data would be necessary, but at present these date are not available.

\section{Description of the sampled sections}

All the sampled sequences pertain to the radiolaritebasalt blocks included in the Ankara Mélange. For the 

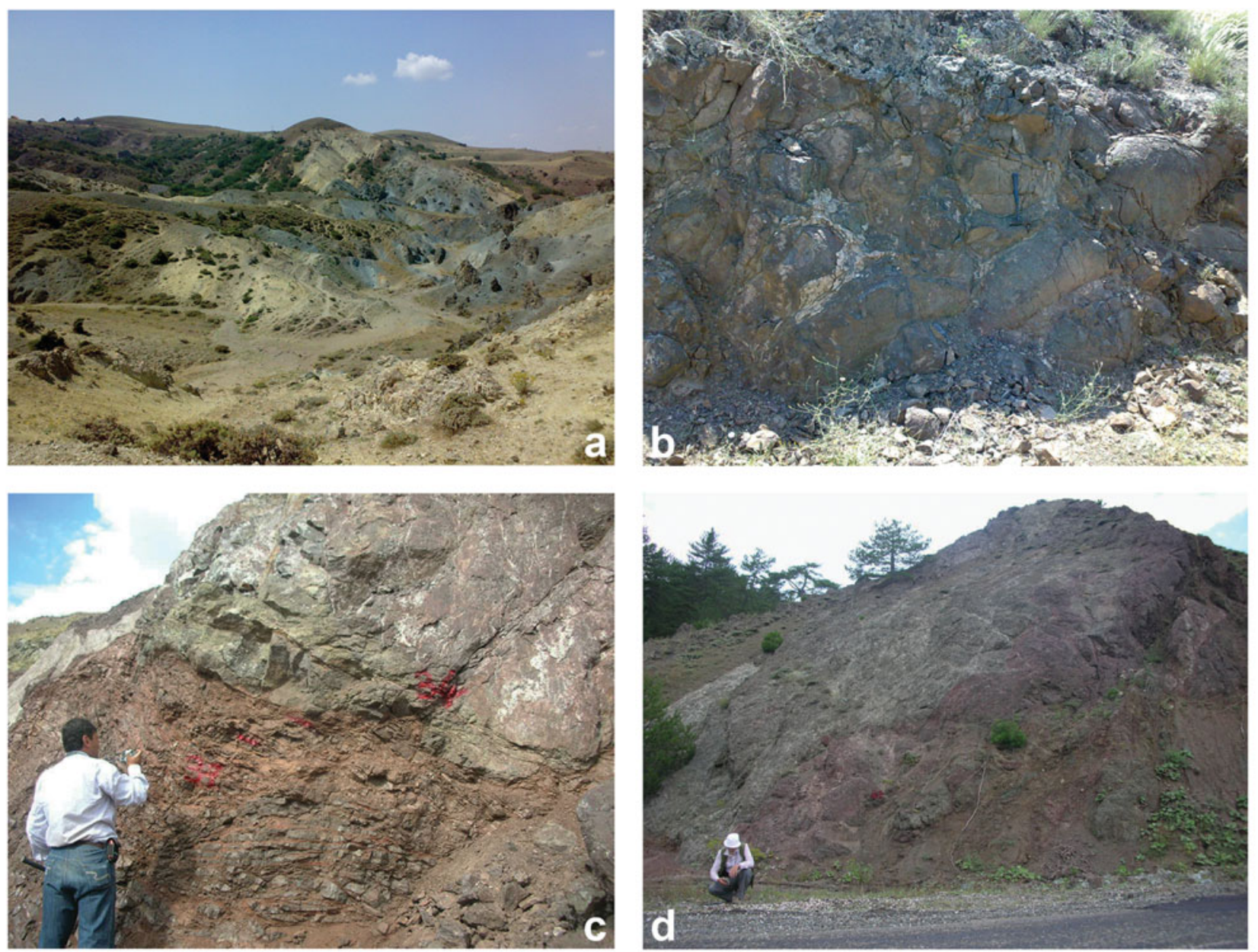

Figure 3. (Colour online) (a) Serpentinite mélange near Beynam. (b) Pillow basalts along the road from Elmadag to Kırıkkale (Section 1). Length of hammer for scale is $32 \mathrm{~cm}$. (c) Overturned sequence of basalts and radiolarian cherts along the road from Çorum to Alaca (Section 5). (d) Outcrop of basalts and radiolarian cherts along the road from Iskilip to Tosya (Section 7). Modified from Bortolotti et al. (2013a).

numbering of the sections we referred to Bortolotti et al. (2013a). It is worth noting that in the present paper we do not report the samples from Section 6 of Late Triassic age of Bortolotti et al. (2013a).

\section{3.a. Section 1}

In a massif of pillow basalts along the ElmadagKirıkkale road, several metric intercalations of siliceous shales with scattered radiolarian cherts crop out.

We sampled one of these intercalations and the associated basalts (Fig. 3b, $39^{\circ} 55.023^{\prime} \mathrm{N}, 33^{\circ} 21.989^{\prime} \mathrm{E}$ ).

Samples: TU10.4, radiolarian chert; TU10.6, TU10.10, basalts below the radiolarian chert intercalation; TU10.9, basalt $\sim 20 \mathrm{~m}$ above the radiolarian chert intercalation.

\section{3.b. Section 2}

In a massif of mainly pillow basalts along the SorgunÇekerek road, thin layers of radiolarian cherts crop out.

We sampled one of these layers and the associated basalts $\left(39^{\circ} 54.889^{\prime} \mathrm{N}, 35^{\circ} 18.063^{\prime} \mathrm{E}\right)$; one radiolarian chert sample was collected along the road in an uncertain stratigraphic position.

Samples: TU10.11, radiolarian chert with uncertain stratigraphic position; TU10.12, radiolarian chert; TU10.14 basalt at the contact with the radiolarites; TU10.15, TU10.16, TU10.17, basalts collected several metres from the radiolarites.

\section{3.c. Section 3}

In a basalt block immediately north of Gökdere village, along the road, a sequence with radiolarian cherts crops out.

Samples: TU10.19, basalt at the contact with the radiolarian cherts $\left(39^{\circ} 59.924^{\prime} \mathrm{N}, 35^{\circ} 24.272^{\prime} \mathrm{E}\right)$; TU10.22, TU10.23, basalts collected some metres southwards $\left(39^{\circ} 59.921^{\prime} \mathrm{N}, 35^{\circ} 24.274^{\prime} \mathrm{E}\right)$, respectively 3.5 and $2 \mathrm{~m}$ from the contact with the radiolarites; TU10.28, radiolarian chert collected $\sim 22 \mathrm{~m}$ from the basalts (this sequence is intensely folded).

\section{3.d. Section 4}

An overturned sequence of basalts and radiolarian cherts with a sheared contact, along the road 
$\sim 3 \mathrm{~km}$ southeast of Bogazkale $\left(40^{\circ} 00.377^{\prime} \mathrm{N}, 34^{\circ}\right.$ $\left.38.762^{\prime} \mathrm{E}\right)$.

Samples: TU10.29, TU10.30, TU10.31, radiolarian cherts collected $\sim 20 \mathrm{~cm}$ above the sheared level; $60 \mathrm{~cm}$ of sheared argillites and cherts separates the basalts from the above samples; TU10.32, basalt at the contact with the sheared level; TU10.33, basalt collected some metres above.

\section{3.e. Section 5}

Big quarry cut by small faults that pull the contact between the basalts-cherts up and down. The sequence could be overturned: the cherts lie under the basalts; the contact is very clear with pockets of argillites and cherts in the mainly massif basalts. The sampled quarry is along the Çorum to Alaca road, south of Küre village (Fig. 3c, $40^{\circ} 15.861^{\prime} \mathrm{N}, 34^{\circ} 48.187^{\prime} \mathrm{E}$ ).

Samples: TU10.34, basalt, sample collected $\sim 6 \mathrm{~m}$ from the cherts; TU10.35, TU10.36, TU10.37, samples collected in a radiolarian chert-argillite sequence from the base to $40 \mathrm{~cm}$ up. A small normal fault shifts the sequence by some metres. TU10.38, radiolarian chert, collected beyond the fault, $\sim 30 \mathrm{~cm}$ from the basalt sample TU10.40; TU10.40, basalt at the contact with the radiolarian cherts; TU10.39, basalt nearby sample TU10.40.

\section{3.f. Section 7}

Small outcrop of basalts and cherts along the IskilipTosya road, $1.2 \mathrm{~km}$ before the junction to Hacihalil village (Fig. 3d, $40^{\circ} 53.378^{\prime} \mathrm{N}, 34^{\circ} 20.756^{\prime} \mathrm{E}$ ).

Samples: TU10.45, radiolarian chert nodule in the basalts; TU10.46, basalt.

\section{3.g. Section 8}

Large body of breccia(?) with basalts, microgabbros, serpentinites and crystallized limestones in a cherty matrix. We sampled the breccia in two close localities northeast of Yukariöz village.

Samples: First locality $\left(40^{\circ} 51.097^{\prime} \mathrm{N}\right.$, $\left.33^{\circ} 50.723^{\prime} \mathrm{E}\right)$, TU10.47, TU10.48, radiolarian cherts; TU10.49a, TU10.49b, basalts. Second locality $\left(40^{\circ} 51.527^{\prime} \mathrm{N}, 33^{\circ} 49.577^{\prime} \mathrm{E}\right)$, TU10.51, M10 radiolarian cherts.

\section{3.h. Section 9}

Pillow basalts enveloped in reddish limestones, Eldivan-Sabanozu road $\left(40^{\circ} 31.488^{\prime} \mathrm{N}, 33^{\circ} 28.106^{\prime} \mathrm{E}\right)$.

Samples: TU10.52, TU10.53, basalts; TU10.54, TU10.55, limestones.

\section{Biostratigraphy}

The radiolarian samples were etched with hydrochloric and hydrofluoric acid following the method proposed by Dumitrica (1970), Pessagno \& Newport (1972),
Baumgartner et al. (1981) and De Wever (1982). The examined samples yielded radiolarians with moderate preservation and the principal marker taxa are illustrated in Figures 4 and 5.

For the taxonomy and ranges of the Late Jurassic - Early Cretaceous principal marker taxa we refer to Aliev (1967), Kozur (1985), Kawabata (1988), Aita \& Okada (1986), Goričan (1994), O’Dogherty (1994), Baumgartner et al. (1995a,b), Dumitrica \& DumitricaJud (1995), Bak (1996, 1999), Dumitrica, Immenhauser \& Dumitrica-Jud (1997), Chiari, Marcucci \& Prela (2004), Danelian et al. (2004), Smuc \& Goričan (2005), Filippov \& Kemkin (2005), Chiari, Cobianchi \& Picotti (2007), Danelian (2008), Dumitrica \& Zügel (2008), O’Dogherty et al. (2009), Robin et al. (2010), Bandini et al. (2011), Goričan, Pavšič \& Rožič (2012) and Chiari et al. (2012).

From the analysed cherts the following radiolarian assemblages and ages were obtained:

\section{4.a. Section 1}

TU10.4. Angulobracchia portmanni Baumgartner, Archaeodictyomitra mitra Dumitrica, Archaeodictyomitra sp. cf. A. lacrimula (Foreman) (Fig. 4a), Archaeodictyomitra sp. cf. A. mitra Dumitrica, Archaeodictyomitra sp., Archaeodictyomitra (?) sp., Aurisaturnalis variabilis variabilis (Squinabol) (Fig. 4b), Hemicryptocapsa sp. cf. H. capita Tan (Fig. 4c), Neorelumbra (?) sp., Pseudodictyomitra sp., Pseudodictyomitra (?) sp., Pyramispongia (?) sp., Tethysetta (?) sp., Thanarla brouweri (Tan) (Fig. 4d), Thanarla sp., Torculum (?) sp., Xitus sp. cf. X. robustus Wu, Xitus sp.

Age. Late Valanginian to late Hauterivian (UAZ. 1720; UAZones after Baumgartner et al. 1995a) based on the presence of Aurisaturnalis variabilis variabilis (Squinabol). Ranges after Baumgartner et al. (1995b).

\section{4.b. Section 2}

TU10.11. Eoxitus (?) sp. (Fig. 4e), Praewilliriedellum sp. cf. P. japonicum (Yao), Stichomitra (?) takanoensis Aita (Fig. 4f), Striatojaponocapsa (?) sp.

Age. Early-middle Bajocian to late Bathonian-early Callovian (UAZ. 3-7; UAZones after Baumgartner et al. 1995a) based on the presence of Stichomitra (?) takanoensis Aita. Range after Baumgartner et al. (1995b).

TU10.12. Hiscocaspsa sp. (Fig. 4g), Mirifusus sp. cf. M. guadalupensis Pessagno (Fig. 4h), Parahsuum sp., Parahsuum (?) sp., Praewilliriedellum sp. cf. $P$. japonicum (Yao), Praewilliriedellum (?) sp., Pseudodictyomitra (?) sp., Stichomitra (?) takanoensis Aita (Fig. 4i), Transhsuum sp., Transhsuum (?) sp., Tritrabs (?) sp.

Age. Early-middle Bajocian to late Bathonian-early Callovian (UAZ. 3-7; UAZones after Baumgartner et al. 1995a) based on the presence of Stichomitra 


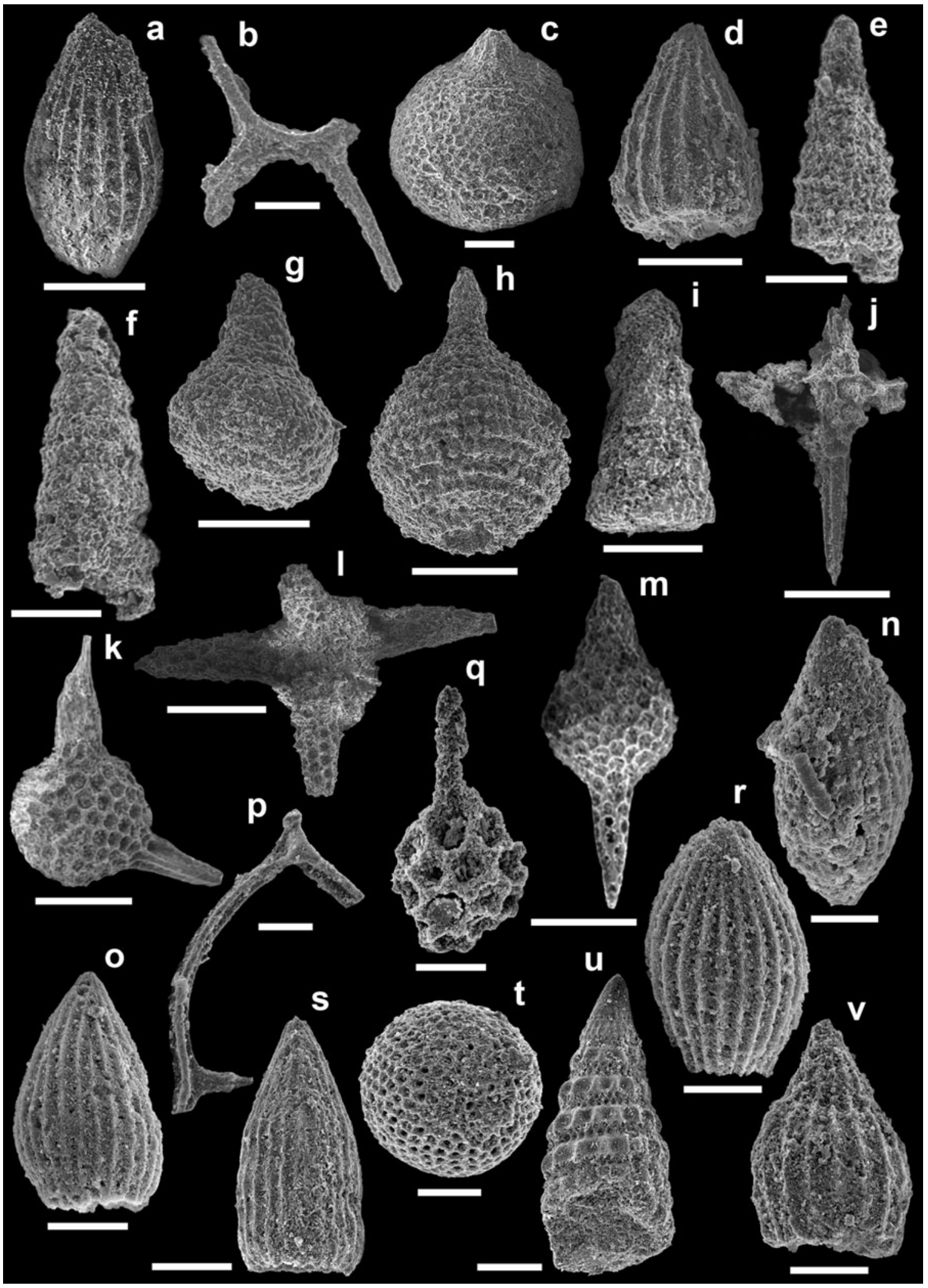

Figure 4. Scale bar $=50 \mu \mathrm{m}$. (a) Archaeodictyomitra sp. cf. A. lacrimula (Foreman), Section 1, TU10.4; (b) Aurisaturnalis variabilis variabilis (Squinabol), Section 1, TU10.4; (c) Hemicryptocapsa sp. cf. H. capita Tan, Section 1, TU10.4; (d) Thanarla brouweri (Tan), Section 1, TU10.4; (e) Eoxitus (?) sp., Section 2, TU10.11; (f) Stichomitra (?) takanoensis Aita, Section 2, TU10.11; (g) Hiscocapsa sp. Section 2, TU10.12; (h) Mirifusus sp. cf. M. guadalupensis Pessagno, Section 2, TU10.12; (i) Stichomitra (?) takanoensis Aita, Section 2, TU10.12; (j) Emiluvia sp. cf. E. ordinaria Ozvoldova, Section 3, TU10.28; (k) Fultacapsa sphaerica (Ozvoldova), Section 3, TU10.28; (1) Podocapsa amphitreptera Foreman, Section 3, TU10.28; (m) Spinosicapsa (?) sp., Section 3, TU10.28; (n) Archaeodictyomitra lacrimula (Foreman), Section 4, TU10.29; (o) Archaeodictyomitra mitra Dumitrica, Section 4, TU10.29; (p) Dicerosaturnalis trizonalis (Rüst), Section 4, TU10.29; (q) Pantanellium sp. cf. P. squinaboli (Tan), Section 4, TU10.29; (r) Thanarla sp. cf. T. gutta Jud, Section 4, TU10.29; (s) Archaeodictyomitra sp., Section 4, TU10.30; (t) Holocryptocanium barbui Dumitrica, Section 4, TU10.30; (u) Pseudodictyomitra lanceloti Schaaf, Section 4, TU10.30; (v) Thanarla sp. cf. T. pacifica Nakaseko \& Nishimura, Section 4, TU10.30. 


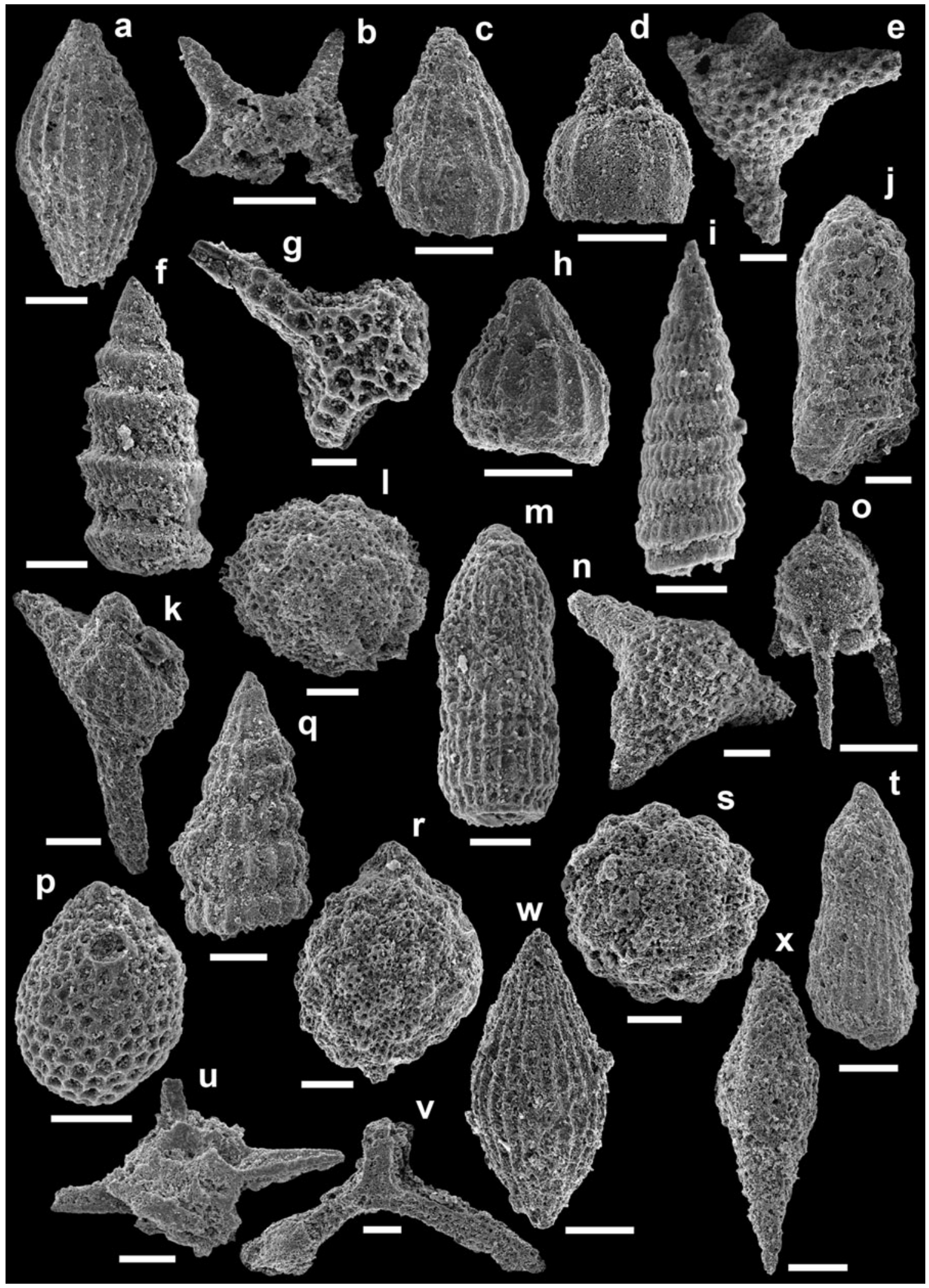

Figure 5. Scale bar $=50 \mu \mathrm{m}$. (a) Archaeodictyomitra lacrimula (Foreman), Section 4, TU10.31; (b) Aurisaturnalis carinatus perforatus Dumitrica \& Dumitrica-Jud, Section 4, TU10.31; (c) Thanarla brouweri (Tan), Section 4, TU10.31; (d) Eucyrtidiellum pyramis (Aita), Section 5, TU10.35; (e) Podocapsa amphitreptera Foreman, Section 5, TU10.35; (f) Cinguloturris cylindra Kemkin \& Rudenko, Section 5, TU10.36; (g) Emiluvia sp. cf. E. ordinaria Ozvoldova, Section 5, TU10.36; (h) Eucyrtidiellum pyramis (Aita), Section 5, TU10.36; (i) Loopus primitivus (Matsuoka \& Yao), Section 5, TU10.36; (j) Ristola cretacea (Baumgartner), Section 5, TU10.36; (k) Podocapsa amphitreptera Foreman, Section 5, TU10.37; (1) Praeconosphaera (?) sphaeroconus (Rüst), Section 5, TU10.37; (m) Archaeodictyomitra sp. cf. A. excellens (Tan), Section 5, TU10.38; (n) Podocapsa amphitreptera Foreman, Section 5, TU10.38; (o) Saitoum sp. cf. S. elegans De Wever, Section 5, TU10.38; (p) Zhamoidellum ovum Dumitrica, Section 5, TU10.38; (q) Archaeodictyomitra sp. cf. A. coniforma Dumitrica, Section 7, TU10.45; (r) Cryptamphorella clivosa (Aliev), Section 7, TU10.45; (s) Praeconosphaera (?) sphaeroconus (Rüst), Section 7, TU10.45; (t) Archaeodictyomitra excellens (Tan), Section 8, TU10.47; (u) Cana septemporatus (Parona), Section 8, TU10.47; (v) Halesium sp. cf. H. palmatum Dumitrica, Section 8, TU10.47; (w) Archaeodictyomitra lacrimula (Foreman), Section 8, TU10.51; (x) Pseudoeucyrtis sp. cf. P. hanni (Tan) sensu O'Dogherty (1994), Section 8, TU10.51. 
(?) takanoensis Aita. Range after Baumgartner et al. (1995b).

\section{4.c. Section 3}

TU.10.28. Acaeniotylopsis sp., Crolanium (?) sp., Emiluvia sp. cf. E. ordinaria Ozvoldova (Fig. 4j), Eoxitus (?) sp., Fultacapsa sphaerica (Ozvoldova) (Fig. 4k), Mirifusus sp. cf. M. guadalupensis Pessagno, Mirifusus sp., Podocapsa amphitreptera Foreman (Fig. 41), Spinosicapsa sp. cf. S. triacantha (Fischli), Spinosicapsa (?) sp. (Fig. 4m), Spongocapsula sp., Svinitzium sp., Svinitzium (?) sp., Triactoma (?) sp.

Age. Middle-late Oxfordian to late Kimmeridgianearly Tithonian (UAZ. 9-11; UAZones after Baumgartner et al. 1995a) based on the occurrence of Podocapsa amphitreptera Foreman with Fultacapsa sphaerica (Ozvoldova). Ranges after Baumgartner et al. (1995b).

\section{4.d. Section 4}

TU10.29. Archaeodictyomitra lacrimula (Foreman) (Fig. 4n), Archaeodictyomitra mitra Dumitrica (Fig. 4o), Archaeodictyomitra sp., Dicerosaturnalis trizonalis (Rüst) (Fig. 4p), Holocryptocanium sp. cf. H. barbui Dumitrica, Orbiculiformella (?) sp., Pantanellium sp. cf. P. squinaboli (Tan) (Fig. 4q), Praeconosphaera (?) sp. cf. P. (?) multiconus Yang, Praeconosphaera (?) sp., Praewilliriedellum sp. cf. P. japonicum (Yao), Pseudoeucyrtis sp., Thanarla brouweri (Tan) sensu O’Dogherty (1994), Thanarla sp. cf. T. gutta Jud (Fig. 4r), Thanarla sp.

Age. Early-early late Berriasian to middle Aptianearly Albian (UAZ. 14 - Costata Subzone of Turbocapsula Zone; zone after O’Dogherty, 1994; UAZone after Baumgartner et al. 1995a) based on the occurrence of Archaeodictyomitra lacrimula (Foreman) and Archaeodictyomitra mitra Dumitrica. Ranges after Baumgartner et al. (1995b), O'Dogherty (1994), Dumitrica, Immenhauser \& Dumitrica-Jud (1997) and Bandini et al. (2011).

TU10.30. Archaeodictyomitra sp. cf. A. communis (Squinabol), Archaeodictyomitra sp. cf. A. lacrimula (Foreman), Archaeodictyomitra sp. (Fig. 4s), Archaeodictyomitra (?) sp., Emiluvia (?) sp., Eoxitus sp., Hiscocapsa (?) sp., Holocryptocanium barbui Dumitrica (Fig. 4t), Holocryptocanium sp. cf. H. barbui Dumitrica, Pantanellium sp., Praeconosphaera (?) sp., Pseudodictyomitra lanceloti Schaaf (Fig. 4u), Pseudodictyomitra sp. cf. P. lanceloti Schaaf, Thanarla sp. aff. T. brouweri (Tan), Thanarla sp. cf. T. pacifica Nakaseko \& Nishimura (Fig. 4v), Thanarla sp. cf. T. praeveneta Pessagno, Thanarla sp., Thanarla (?) sp.

Age. Late Hauterivian to middle Aptian-early Albian (UAZ. 20 - Costata Subzone of Turbocapsula Zone; zone after O'Dogherty, 1994; UAZone after Baumgartner et al. 1995a) based on the presence of Pseudodictyomitra lanceloti Schaaf. Range after Baumgartner et al. (1995b) and Bandini et al. (2011).
TU10.31. Angulobracchia (?) sp., Archaeodictyomitra lacrimula (Foreman) (Fig. 5a), Archaeodictyomitra mitra Dumitrica, Archaeodictyomitra sp. cf. A. lacrimula (Foreman), Archaeodictyomitra sp., Aurisaturnalis carinatus perforatus Dumitrica \& Dumitrica-Jud (Fig. 5b), Dicerosaturnalis trizonalis (Rüst), Halesium (?) sp., Pantanellium sp., Praeconosphaera (?) sp. cf. P. (?) multiconus Yang, Praeconosphaera (?) sp., Praexitus sp., Pseudodictyomitra lanceloti Schaaf, Pseudodictyomitra sp. cf. P. lanceloti Schaaf, Pseudodictyomitra sp., Pseudodictyomitra (?) sp., Spinosicapsa (?) sp., Thanarla brouweri (Tan) (Fig. 5c), Thanarla sp. cf. T. brouweri (Tan), Thanarla sp. cf. T. pacifica Nakaseko \& Nishimura, Thanarla sp. cf. T. pulchra (Squinabol), Thanarla sp., Xitus sp., Xitus (?) sp.

Age. Middle late Barremian-early early Aptian based on the occurrence of Aurisaturnalis carinatus perforatus Dumitrica \& Dumitrica-Jud. Range after Dumitrica \& Dumitrica-Jud (1995).

\section{4.e. Section 5}

TU10.35. Archaeodictyomitra (?) sp., Dicerosaturnalis trizonalis (Rüst), Dicerosaturnalis sp. cf. D. trizonalis (Rüst), Emiluvia sp. cf. E. ultima Baumgartner, Emiluvia sp., Eucyrtidiellum pyramis (Aita) (Fig. 5d), Mirifusus sp. cf. M. dianae (Karrer) s.1., Mirifusus sp., $\mathrm{Na}$ pora sp., Podocapsa amphitreptera Foreman (Fig. 5e), Praeconosphaera (?) sp., Pseudodictyomitra (?) sp., Spinosicapsa (?) sp., Svinitzium (?) sp., Syringocapsa (?) sp., Triactoma sp. cf. T. jonesi (Pessagno), Triactoma (?) sp., Zhamoidellum (?) sp.

Age. Early-early late Tithonian to latest Tithonianearliest Berriasian (UAZ. 12-13; UAZones after Baumgartner et al. 1995a) based on the presence of Eucyrtidiellum pyramis (Aita). Range after Baumgartner et al. (1995b).

TU10.36. Archaeodictyomitra apiarium (Rüst), Archaeodictyomitra sp. aff. A. excellens (Tan), Archaeodictyomitra sp. aff. A. rigida Pessagno, Archaeodictyomitra sp. cf. A. apiarium (Rüst), Archaeodictyomitra sp. 1, Archaeodictyomitra sp., Cinguloturris cylindra Kemkin \& Rudenko (Fig. 5f), Emiluvia sp. cf. E. ordinaria Ozvoldova (Fig. 5g), Emiluvia sp. cf. E. orea Baumgartner, Emiluvia (?) sp., Eucyrtidiellum pyramis (Aita) (Fig. 5h), Eucyrtidiellum (?) sp. cf. E. (?) quinatum Takemura, Loopus primitivus (Matsuoka \& Yao) (Fig. 5i), Loopus sp. cf. L. doliolum Dumitrica, Mirifusus dianae s.l. (Karrer), Mirifusus sp., Olanda sp., Podocapsa amphitreptera Foreman, Praeconosphaera (?) sphaeroconus (Rüst), Praeconosphaera (?) sp. cf. P. (?) sphaeroconus (Rüst), Praeconosphaera (?) sp., Pseudodictyomitra sp. cf. P. carpatica (Lozyniak), Pseudodictyomitra (?) sp., Ristola cretacea (Baumgartner) (Fig. 5j), Ristola (?) sp., Sethocapsa horokanaiensis Kawabata, Syringocapsa (?) sp., Zhamoidellum sp. cf. Z. sp. A sensu Goričan (1994), Zhamoidellum sp. 
Age. Early-early late Tithonian (UAZ. 12; UAZone after Baumgartner et al. 1995a) based on the occurrence of Cinguloturris cylindra Kemkin \& Rudenko, Eucyrtidiellum pyramis (Aita) and Ristola cretacea (Baumgartner) with Loopus primitivus (Matsuoka \& Yao). Ranges after Baumgartner et al. (1995b).

TU10.37. Archaeodictyomitra (?) sp., Podocapsa amphitreptera Foreman (Fig. 5k), Praeconosphaera (?) sphaeroconus (Rüst) (Fig. 51), Pseudodictyomitra (?) sp., Spinosicapsa (?) sp.

Age. Late Oxfordian-early Kimmeridgian to latest Valanginian-earliest Hauterivian (UAZ. 10-18; UAZones after Baumgartner et al. 1995a) based on the occurrence of Praeconosphaera (?) sphaeroconus (Rüst) with Podocapsa amphitreptera Foreman. Ranges after Baumgartner et al. (1995b), Chiari, Cobianchi \& Picotti (2007) and Bandini et al. (2011).

TU10.38. Archaeodictyomitra sp. aff. A. chalilovi (Aliev), Archaeodictyomitra sp. aff. A. excellens (Tan), Archaeodictyomitra sp. aff. A. ioniana Danelian, Archaeodictyomitra sp. cf. A. apiarium (Rüst), Archaeodictyomitra sp. cf. A. excellens (Tan) (Fig. 5m), Archaeodictyomitra sp. cf. A. minoensis (Mizutani), Archaeodictyomitra sp., Archaeodictyomitra (?) sp., Becus (?) sp., Cinguloturris sp. cf. C. carpatica Dumitrica, Dicerosaturnalis trizonalis (Rüst), Dicerosaturnalis sp. cf. D. trizonalis (Rüst), Emiluvia sp., Pantanellium sp., Podocapsa amphitreptera Foreman (Fig. 5n), Praeconosphaera (?) sp., Pseudodictyomitra sp. cf. P. thurowi Dumitrica, Pseudodictyomitra sp., Saitoum sp. cf. S. elegans De Wever (Fig. 5o), Saitoum (?) sp., Spinosicapsa (?) sp., Syringocapsa (?) sp., Zhamoidellum ovum Dumitrica (Fig. 5p), Zhamoidellum sp.

Age. Middle-late Oxfordian to late Kimmeridgianearly Tithonian (UAZ. 9-11; UAZones after Baumgartner et al. 1995a) based on the presence of Podocapsa amphitreptera Foreman and Zhamoidellum ovum Dumitrica. Ranges after Baumgartner et al. (1995b) and Smuc \& Goričan (2005).

\section{4.f. Section 7}

TU10.45. Archaeodictyomitra sp. cf. A. communis (Squinabol), Archaeodictyomitra sp. cf. A. coniforma Dumitrica (Fig. 5q), Archaeodictyomitra sp., Archaeodictyomitra (?) sp., Cryptamphorella clivosa (Aliev) (Fig. 5r), Godia sp. cf. G. decora (Li \& Wu), Godia (?) sp., Holocryptocanium sp. cf. H. barbui Dumitrica, Praeconosphaera (?) sphaeroconus (Rüst) (Fig. 5s), Praeconosphaera (?) sp., Pseudodictyomitra (?) sp., Tethysetta sp. cf. T. usotanensis (Tumanda), Thanarla brouweri (Tan), Thanarla sp. cf. T. pulchra (Squinabol), Thanarla sp., Thanarla (?) sp.

Age. Valanginian to middle Aptian-early Albian (Valanginian to Costata Subzone of Turbocapsula Zone; zone after O'Dogherty, 1994) based on the presence of Cryptamphorella clivosa (Aliev) with Praeconosphaera (?) sphaeroconus (Rüst). Ranges after Aliev (1967), O’Dogherty (1994), Filippov \&
Kemkin (2005), Chiari, Cobianchi \& Picotti (2007) and Bandini et al. (2011).

\section{4.g. Section 8}

TU10.47. Archaeodictyomitra excellens (Tan) (Fig. 5t), Archaeodictyomitra lacrimula (Foreman), Archaeodictyomitra sp. cf. A. communis (Squinabol), Archaeodictyomitra sp. cf. A. ioniana Danelian, Archaeodictyomitra sp. cf. A. vulgaris Pessagno, Archaeodictyomitra sp., Archaeodictyomitra (?) sp., Cana septemporatus (Parona) (Fig. 5u), Halesium sp. cf. H. palmatum Dumitrica (Fig. 5v), Hiscocapsa sp. cf. H. grutterinki (Tan), Hiscocapsa sp., Holocryptocanium barbui Dumitrica, Holocryptocanium sp. cf. H. barbui Dumitrica, Pantanellium sp., Praeconosphaera (?) sp. cf. P. (?) sphaeroconus (Rüst), Praeconosphaera (?) sp., Pseudodictyomitra sp. cf. P. sp. 5 in Dumitrica et al. (1997), Pseudodictyomitra sp., Pseudodictyomitra (?) sp., Pseudoxitus (?) sp., Syringocapsa (?) sp., Thanarla sp. cf. T. brouweri (Tan), Thanarla sp., Thanarla (?) sp., Williriedellum (?) sp., Xitus sp. cf. X. normalis (Wu \& Li), Xitus sp., Xitus (?) sp.

Age. Late Valanginian-early Barremian (UAZ. 1721; UAZones after Baumgartner et al. 1995a) based on the presence of Cana septemporatus (Parona). Ranges after Baumgartner et al. (1995b).

TU10.48. Age not determinable because of the very low preservation of radiolarians.

TU10.51. Acaeniotyle sp. cf. A. umbilicata (Rüst), Archaeodictyomitra lacrimula (Foreman) (Fig. $5 \mathrm{w}$ ), Archaeodictyomitra sp. cf. A. communis (Squinabol), Archaeodictyomitra sp., Archaeodictyomitra (?) sp., Crucella (?) sp., Praeconosphaera (?) sp., Pseudodictyomitra (?) sp., Pseudoeucyrtis sp. cf. P. hanni (Tan) sensu O'Dogherty (1994) (Fig. 5x), Spinosicapsa (?) sp., Thanarla sp. cf. T. pulchra (Squinabol), Thanarla sp.

Age. Early-early late Berriasian to middle Aptianearly Albian (UAZ. 14 - Costata Subzone of Turbocapsula Zone; zone after O'Dogherty, 1994; UAZone after Baumgartner et al. 1995a) based on the presence of Archaeodictyomitra lacrimula (Foreman). Range after Baumgartner et al. (1995b) and O’Dogherty (1994).

TU10.M10. Archaeodictyomitra sp., Archaeodictyomitra (?) sp., Kilinora (?) sp., Triversus (?) sp.

Age. Age not determinable because of the low preservation of radiolarians.

\section{Petrography and geochemistry of the basaltic rocks}

\section{5.a. Petrography}

All the studied rocks are affected by low-temperature, ocean-floor alteration, which resulted in the replacement of primary minerals, though primary igneous textures are well preserved. Plagioclase is 
usually replaced by albite or calcite and rarely by clay mineral assemblages. Clinopyroxene is normally pseudomorphosed either by chlorite or actinolitic amphibole. In samples TU10.22 and TU10.23 (Section 3) clinopyroxene is replaced by brown hornblende, though fresh clinopyroxene relics are locally observed. The groundmass secondary phases mainly consist of chlorite and clay minerals. Regardless of the secondary mineralogical transformation, the following petrographic description of the various rock types has been made on the basis of the primary igneous phases. Moreover, for a better understanding, it has been made according to the geochemical groups described below.

Group 1. Pillow and massive lavas have aphyric, micro-crystalline sub-ophitic textures in which only small laths of plagioclase can be recognized. Pillow breccias are generally monogenetic and show a coarsegrained, intergranular texture with euhedral plagioclase and interstitial clinopyroxene.

Group 2. Massive lavas show both aphyric and porphyritic (PI $=\sim 40)$ textures. In both varieties, the groundmass texture is hyalopilitic. Phenocrysts are represented by large crystals of plagioclase.

Groups 3 and 4. Pillow and massive lavas most commonly display aphyric, ophitic or sub-ophitic textures with crystal sizes ranging from micro-crystalline to coarse grained. Nonetheless, a few samples display slightly porphyritic textures with plagioclase microphenocrysts. In addition, a hyalopilitic texture is locally observed. The groundmass mineral assemblage includes plagioclase, clinopyroxene and variable amounts of opaque phases. Pillow breccias are generally monogenetic and the individual fragments are texturally and compositionally similar to the pillow lavas. Most of these rocks are characterized by variable abundances of varioles filled by calcite. In all the studied rock groups, the crystallization order is: plagioclase + clinopyroxene $\pm \mathrm{Fe}$-Ti-oxides.

\section{5.b. Analytical methods}

Whole-rock major- and some trace-element analyses were obtained by X-ray fluorescence (XRF) on pressed-powder pellets, using an ARL Advant-XP automated X-ray spectrometer. The matrix correction methods proposed by Lachance \& Trail (1966) were applied. Volatile contents were determined as loss on ignition (LOI) at $1000^{\circ} \mathrm{C}$. In addition, $\mathrm{Rb}, \mathrm{Sr}, \mathrm{Nb}, \mathrm{Hf}$, $\mathrm{Ta}, \mathrm{Th}, \mathrm{U}$ and the rare earth elements (REEs) were determined on 12 representative samples by inductively coupled plasma-mass spectrometry (ICP-MS) using a Thermo Series X-I spectrometer.

The $\mathrm{CO}_{2}$ content was determined by the simple volumetric technique (Jackson, 1958) only on the samples affected by calcite veins and amygdales. This technique was calibrated using standard amounts of reagent grade $\mathrm{CaCO}_{3}$. In addition, for the discussion of the geochemical characteristics and for a better comparison of chemical data, the major-element compositions of these samples were recalculated on a calcite-free basis. In detail, $\mathrm{CaO}$ content in secondary calcite has been calculated according to stoikiometric proportions with $\mathrm{CO}_{2}$ contents, given that the secondary carbonates are exclusively composed of calcite. Major-element composition has then been recalculated to $100 \mathrm{wt} \%$ without considering $\mathrm{LOI}$ and $\mathrm{CaO}$ in calcite.

The accuracy of the data for XRF and ICP-MS analyses were evaluated using results for international standard rocks run as unknowns. The detection limits for XRF and ICP-MS analyses were evaluated using results from several runs of $\sim 29$ international standards. Accuracy and detection limits for the $\mathrm{CO}_{2}$, analyses were determined using different amounts of reagent grade $\mathrm{CaCO}_{3}$ run as unknowns. Results are given in the online Supplementary Material available at http://journals.cambridge.org/geo. All whole-rock analyses were performed at the Dipartimento di Science della Terra, Università di Ferrara. The results are shown in Table 1.

\section{5.c. Geochemistry}

The following geochemical description is made mainly using those major and trace elements that are virtually immobile during low-temperature alteration and metamorphism (e.g. Pearce \& Norry, 1979). These elements include many incompatible elements, such as Ti, $\mathrm{P}, \mathrm{Zr}, \mathrm{Y}, \mathrm{Sc}, \mathrm{Nb}, \mathrm{Ta}, \mathrm{Hf}, \mathrm{Th}$, middle (M-) and heavy (H-) REEs, as well as some transition metals (e.g. $\mathrm{Ni}, \mathrm{Co}, \mathrm{Cr}, \mathrm{V}$ ). Light REEs (LREEs) may be affected by some mobilization during alteration. However, the good correlations between these elements and many immobile elements (not shown) indicate that LREEs have not been mobilized by the alteration. For example, the correlation coefficients $\left(\mathrm{r}^{2}\right)$ for the linear correlation $\mathrm{Zr}-\mathrm{La}$ and $\mathrm{Zr}-\mathrm{Ce}$ are 0.96 and 0.90 , respectively. $\mathrm{Rb}, \mathrm{Ba}, \mathrm{K}$ and $\mathrm{Sr}$ are commonly mobilized during alteration. In fact, these elements plotted against $\mathrm{Zr}$ generally show low or very low $\mathrm{r}^{2}$. However, the fairly good correlation with $\mathrm{Zr}$ and $\mathrm{Rb}$ in Group 4 samples $\left(r^{2}=0.91\right)$, as well as Ba in Group $4\left(r^{2}=0.87\right)$ and in Group 2 samples $\left(r^{2}=0.85\right)$, suggests that these elements were only slightly mobilized during alteration in these rock types.

According to Bortolotti et al. (2013a), four groups of volcanic rocks can be recognized in the studied sections. Group 1 is represented by basalts cropping out in Sections 5 and 8. These rocks have a clear sub-alkaline nature with low $\mathrm{Nb} / \mathrm{Y}$ ratios (Fig. 6) and show relatively high $\mathrm{TiO}_{2}, \mathrm{P}_{2} \mathrm{O}_{5}, \mathrm{Zr}$ and $\mathrm{Y}$ contents. $\mathrm{Ni}$ is generally low, whereas $\mathrm{Cr}$ is relatively high, with the only exception of sample TU10.49b. V content is also generally high (Table 1). The values of these elements are similar to those of Group 2 basalts (see below). By contrast, Hf, Ta, Th and U contents are low. These rocks show a sharp increase in $\mathrm{FO}_{\mathrm{t}}$ and $\mathrm{Y}$ and a sharp decrease in $\mathrm{Mg}$ no. and Ni with increasing $\mathrm{Zr}$, as well as very low $\mathrm{Th} / \mathrm{Tb}$ ratios (Fig. 7). Group 1 basalts are characterized by flat normalized incom- 
Table 1. Representative major- and trace-element analyses of Middle Jurassic - Early Cretaceous volcanic rocks from the Ankara Mélange

\begin{tabular}{|c|c|c|c|c|c|c|c|c|c|c|}
\hline \multirow{2}{*}{$\begin{array}{l}\text { Section } \\
\text { Sample }\end{array}$} & \multicolumn{3}{|c|}{1} & \multicolumn{4}{|c|}{2} & \multicolumn{3}{|c|}{3} \\
\hline & TU10.6 & TU10.9 & TU10.10 & TU10.14 & TU10.15 & TU10.16 & TU10.17 & TU10.19 & TU10.22 & TU10.23 \\
\hline Rock & bas & tra & bas & bas & bas & $\mathrm{Fe}-\mathrm{bas}$ & Fe-bas & bas & bas & bas \\
\hline Group & 4 & 4 & 4 & 3 & 3 & 3 & 3 & 4 & 4 & 4 \\
\hline \multirow[t]{2}{*}{ Type } & alkaline & alkaline & alkaline & trans & trans & trans & trans & alkaline & alkaline & alkaline \\
\hline & OIB & OIB & OIB & P-MORB & P-MORB & P-MORB & P-MORB & OIB & OIB & OIB \\
\hline Age & $\mathrm{E} \mathrm{Cr}$ & & & M Jr & M Jr & M Jr & $\mathrm{M} \mathrm{Jr}$ & & $\mathrm{L} \mathrm{Jr}$ & $\mathrm{L} \mathrm{Jr}$ \\
\hline \multirow[t]{2}{*}{ Note } & pillow & breccia & pillow & pillow & pillow & pillow & pillow & mlf & pillow & pillow \\
\hline & $(X R F)$ & $(X R F)$ & $(X R F)$ & $(X R F)$ & $(X R F)$ & $(X R F)$ & $(X R F)$ & $(X R F)$ & $(X R F)$ & $(X R F)$ \\
\hline $\mathrm{SiO}_{2}$ & 41.40 & 56.12 & 39.61 & 44.12 & 40.20 & 42.40 & 44.87 & 44.36 & 46.03 & 44.81 \\
\hline $\mathrm{TiO}_{2}$ & 1.91 & 2.35 & 1.96 & 2.28 & 1.65 & 3.27 & 2.55 & 2.97 & 2.32 & 2.21 \\
\hline $\mathrm{Al}_{2} \mathrm{O}_{3}$ & 11.76 & 15.96 & 11.81 & 14.48 & 11.92 & 13.81 & 13.82 & 13.41 & 17.06 & 16.49 \\
\hline $\mathrm{Fe}_{2} \mathrm{O}_{3}$ & 0.93 & 1.33 & 1.00 & 1.74 & 1.00 & 2.08 & 1.96 & 1.45 & 1.42 & 1.40 \\
\hline $\mathrm{FeO}$ & 6.18 & 8.90 & 6.67 & 11.60 & 6.66 & 13.89 & 13.06 & 9.63 & 9.47 & 9.31 \\
\hline $\mathrm{MnO}$ & 0.15 & 0.11 & 0.12 & 0.23 & 0.22 & 0.19 & 0.19 & 0.40 & 0.20 & 0.20 \\
\hline $\mathrm{MgO}$ & 4.24 & 1.68 & 3.86 & 9.25 & 6.32 & 7.42 & 9.98 & 9.14 & 7.31 & 9.05 \\
\hline $\mathrm{CaO}$ & 16.69 & 3.61 & 17.84 & 8.16 & 18.02 & 8.04 & 6.23 & 6.96 & 6.48 & 7.45 \\
\hline $\mathrm{Na}_{2} \mathrm{O}$ & 3.73 & 7.37 & 2.93 & 2.39 & 2.11 & 3.07 & 1.74 & 3.24 & 2.07 & 1.84 \\
\hline $\mathrm{K}_{2} \mathrm{O}$ & 1.72 & 0.65 & 2.06 & 0.82 & 1.89 & 0.69 & 1.26 & 1.13 & 3.54 & 2.95 \\
\hline $\mathrm{P}_{2} \mathrm{O}_{5}$ & 0.48 & 0.59 & 0.60 & 0.33 & 0.21 & 0.54 & 0.62 & 0.52 & 0.96 & 0.86 \\
\hline L.O.I. & 10.94 & 1.16 & 11.56 & 4.65 & 9.89 & 4.65 & 3.73 & 6.94 & 3.15 & 3.50 \\
\hline Total & 100.13 & 99.84 & 100.03 & 100.05 & 100.08 & 100.05 & 100.01 & 100.15 & 100.01 & 100.06 \\
\hline $\mathrm{CO}_{2}$ & 6.23 & & 6.13 & & 7.05 & & & & & \\
\hline Mg no. & 55.0 & 25.2 & 50.8 & 58.7 & 62.9 & 48.8 & 57.7 & 62.8 & 57.9 & 63.4 \\
\hline $\mathrm{Zn}$ & 73 & 104 & 57 & 72 & 63 & 110 & 101 & 109 & 75 & 72 \\
\hline $\mathrm{Cu}$ & 25 & 35 & 44 & 77 & 71 & 59 & 57 & 69 & 40 & 40 \\
\hline $\mathrm{Sc}$ & 23 & 9 & 18 & 36 & 40 & 43 & 35 & 36 & 8 & 10 \\
\hline $\mathrm{Ga}$ & 15 & 14 & 16 & 21 & 15 & 24 & 20 & 16 & 17 & 19 \\
\hline $\mathrm{Ni}$ & 70 & 26 & 54 & 78 & 53 & 8 & 11 & 100 & 25 & 42 \\
\hline $\mathrm{Co}$ & 27 & 16 & 23 & 37 & 33 & 37 & 30 & 36 & 23 & 25 \\
\hline $\mathrm{Cr}$ & 227 & 56 & 115 & 135 & 81 & 26 & 21 & 271 & 16 & 29 \\
\hline V & 204 & 180 & 206 & 334 & 275 & 403 & 329 & 474 & 188 & 210 \\
\hline $\mathrm{Ba}$ & 289 & 186 & 340 & 246 & 717 & 199 & 177 & 193 & 1980 & 1380 \\
\hline $\mathrm{Pb}$ & 4 & 7 & 5 & 7 & 4 & 7 & 6 & 16 & 8 & 7 \\
\hline $\mathrm{Zr}$ & 217 & 320 & 225 & 165 & 128 & 239 & 196 & 265 & 330 & 287 \\
\hline & $(I C P-M S)$ & & $(I C P-M S)$ & $(I C P-M S)$ & $(I C P-M S)$ & & & $(I C P-M S)$ & & $(I C P-M S)$ \\
\hline $\mathrm{Rb}$ & 30.8 & 12.0 & 36.7 & 8.41 & 17.6 & 8.0 & 16.0 & 27.2 & 75.0 & 58.5 \\
\hline $\mathrm{Sr}$ & 575 & 441 & 478 & 292 & 280 & 391 & 371 & 197 & 740 & 760 \\
\hline Y & 28.5 & 40.0 & 29.3 & 22.0 & 27.6 & 58.0 & 48.0 & 29.0 & 39.0 & 36.1 \\
\hline $\mathrm{La}$ & 40.3 & 64.0 & 39.0 & 20.7 & 24.6 & 50.0 & 45.0 & 49.7 & 65.0 & 58.9 \\
\hline $\mathrm{Ce}$ & 70.3 & 192.0 & 70.9 & 43.0 & 46.7 & 129.0 & 82.0 & 85.3 & 160.0 & 109.0 \\
\hline $\operatorname{Pr}$ & 8.01 & & 8.14 & 4.91 & 5.35 & & & 9.25 & & 11.3 \\
\hline $\mathrm{Nd}$ & 27.2 & 45.0 & 28.1 & 18.1 & 19.1 & 33.0 & 30.0 & 30.4 & 60.0 & 39.7 \\
\hline $\mathrm{Sm}$ & 5.84 & & 6.07 & 4.63 & 4.54 & & & 6.11 & & 9.08 \\
\hline $\mathrm{Eu}$ & 1.77 & & 1.84 & 1.53 & 1.53 & & & 1.69 & & 2.77 \\
\hline $\mathrm{Gd}$ & 4.50 & & 4.69 & 4.60 & 4.45 & & & 4.62 & & 7.11 \\
\hline $\mathrm{Tb}$ & 0.702 & & 0.722 & 0.718 & 0.657 & & & 0.697 & & 1.03 \\
\hline Dy & 3.71 & & 3.76 & 4.28 & 3.82 & & & 3.67 & & 5.33 \\
\hline Но & 0.704 & & 0.711 & 0.896 & 0.793 & & & 0.707 & & 0.992 \\
\hline $\mathrm{Er}$ & 1.78 & & 1.78 & 2.38 & 2.11 & & & 1.81 & & 2.58 \\
\hline $\mathrm{Tm}$ & 0.239 & & 0.236 & 0.339 & 0.295 & & & 0.242 & & 0.35 \\
\hline $\mathrm{Yb}$ & 1.49 & & 1.49 & 2.21 & 1.92 & & & 1.54 & & 2.19 \\
\hline $\mathrm{Lu}$ & 0.214 & & 0.213 & 0.335 & 0.291 & & & 0.221 & & 0.324 \\
\hline $\mathrm{Nb}$ & 61.5 & 83.0 & 65.9 & 30.6 & 40.4 & 50.0 & 45.0 & 88.0 & 142.0 & 88.0 \\
\hline $\mathrm{Hf}$ & 5.11 & 5.00 & 5.34 & 4.56 & 3.69 & 4.00 & 3.00 & 4.82 & 5.00 & 7.00 \\
\hline $\mathrm{Ta}$ & 2.93 & & 3.71 & 1.76 & 3.34 & & & 4.77 & & 7.13 \\
\hline $\mathrm{Th}$ & 5.09 & 9.00 & 5.49 & 2.77 & 3.48 & 7.00 & 5.00 & 7.49 & 9.00 & 11.6 \\
\hline $\mathrm{U}$ & 1.48 & & 1.34 & 0.793 & 1.04 & & & 1.75 & & 2.99 \\
\hline$(\mathrm{La} / \mathrm{Sm})_{\mathrm{N}}$ & 4.46 & & 4.15 & 2.88 & 3.49 & & & 5.25 & & 4.19 \\
\hline$(\mathrm{Sm} / \mathrm{Yb})_{\mathrm{N}}$ & 4.36 & & 4.52 & 2.33 & 2.63 & & & 4.40 & & 4.62 \\
\hline$(\mathrm{La} / \mathrm{Yb})_{\mathrm{N}}$ & 19.43 & & 18.75 & 6.72 & 9.17 & & & 23.13 & & 19.33 \\
\hline $\mathrm{Ti} / \mathrm{V}$ & 69 & 79 & 71 & 43 & 44 & 51 & 48 & 40 & 76 & 65 \\
\hline $\mathrm{Ce} / \mathrm{Y}$ & 2.40 & 4.80 & 2.69 & 1.87 & 1.64 & 2.20 & 1.60 & 3.16 & 4.10 & 4.01 \\
\hline $\mathrm{Nb} / \mathrm{Yb}$ & 41.32 & & 44.13 & 13.86 & 21.03 & & & 57.02 & & 40.25 \\
\hline$(\mathrm{Th} / \mathrm{Ta}) /(\mathrm{Th} / \mathrm{Tb})$ & 0.24 & & 0.19 & 0.41 & 0.20 & & & 0.15 & & 1.14 \\
\hline
\end{tabular}


Table 1. Continued

\begin{tabular}{|c|c|c|c|c|c|c|c|c|c|c|}
\hline \multirow{2}{*}{$\begin{array}{l}\text { Section } \\
\text { Sample }\end{array}$} & \multicolumn{2}{|c|}{4} & \multicolumn{3}{|c|}{5} & \multirow{2}{*}{$\begin{array}{c}7 \\
\text { TU10.46 }\end{array}$} & \multicolumn{2}{|c|}{8} & \multicolumn{2}{|c|}{9} \\
\hline & TU10.32 & TU10.33 & TU10.34 & TU10.39 & TU10.40 & & TU10.49a & TU10.49b & TU10.52 & TU10.53 \\
\hline Rock & bas & bas & bas & bas & bas & bas & bas & bas & bas & bas \\
\hline Group & 2 & 2 & 1 & 1 & 1 & 2 & 1 & 1 & 3 & 3 \\
\hline Type & sub-alk & sub-alk & sub-alk & sub-alk & sub-alk & sub-alk & sub-alk & sub-alk & trans & trans \\
\hline E-MORB & E-MORB & G-MORB & G-MORB & G-MORB & E-MORB & G-MORB & G-MORB & P-MORB & P-MORB & \\
\hline Age & $\mathrm{E} \mathrm{Cr}$ & $\mathrm{E} \mathrm{Cr}$ & $\mathrm{L} \mathrm{Jr}$ & $\mathrm{L} \mathrm{Jr}$ & $\mathrm{L} \mathrm{Jr}$ & $\mathrm{E} \mathrm{Cr}$ & $\mathrm{E} \mathrm{Cr}$ & $\mathrm{E} \mathrm{Cr}$ & & \\
\hline Note & mlf & mlf & mlf & breccia & mlf & mlf & pillow & pillow & mlf & mlf \\
\hline$(X R F)$ & $(X R F)$ & $(X R F)$ & $(X R F)$ & $(X R F)$ & $(X R F)$ & $(X R F)$ & $(X R F)$ & $(X R F)$ & $(X R F)$ & \\
\hline $\mathrm{SiO}_{2}$ & 45.98 & 46.18 & 47.04 & 48.28 & 46.35 & 48.35 & 53.76 & 48.67 & 48.72 & 39.89 \\
\hline $\mathrm{TiO}_{2}$ & 1.51 & 1.46 & 1.48 & 1.46 & 1.74 & 1.20 & 1.51 & 1.46 & 1.89 & 1.37 \\
\hline $\mathrm{Al}_{2} \mathrm{O}_{3}$ & 13.44 & 13.30 & 13.50 & 14.84 & 15.70 & 17.65 & 13.26 & 13.83 & 17.70 & 11.71 \\
\hline $\mathrm{Fe}_{2} \mathrm{O}_{3}$ & 1.44 & 1.26 & 1.28 & 1.42 & 1.59 & 0.77 & 1.28 & 1.65 & 1.42 & 1.05 \\
\hline $\mathrm{FeO}$ & 9.61 & 8.43 & 8.51 & 9.49 & 10.60 & 5.14 & 8.52 & 11.01 & 9.49 & 6.97 \\
\hline $\mathrm{MnO}$ & 0.29 & 0.27 & 0.26 & 0.19 & 0.11 & 0.11 & 0.13 & 0.16 & 0.05 & 0.17 \\
\hline $\mathrm{MgO}$ & 5.69 & 5.14 & 5.24 & 5.48 & 2.40 & 4.69 & 5.80 & 7.48 & 3.77 & 4.26 \\
\hline $\mathrm{CaO}$ & 14.15 & 15.60 & 15.85 & 9.28 & 12.16 & 9.55 & 8.03 & 6.18 & 4.16 & 21.76 \\
\hline $\mathrm{Na}_{2} \mathrm{O}$ & 2.82 & 3.21 & 3.27 & 3.39 & 3.77 & 5.71 & 3.88 & 2.49 & 2.04 & 1.88 \\
\hline $\mathrm{K}_{2} \mathrm{O}$ & 0.46 & 0.40 & 0.41 & 1.49 & 1.31 & 0.29 & 0.36 & 0.88 & 4.64 & 0.53 \\
\hline $\mathrm{P}_{2} \mathrm{O}_{5}$ & 0.14 & 0.13 & 0.14 & 0.17 & 0.28 & 0.12 & 0.17 & 0.11 & 0.32 & 0.14 \\
\hline L.O.I. & 4.58 & 4.66 & 3.02 & 4.56 & 3.94 & 6.45 & 3.2 & 6.02 & 5.83 & 10.28 \\
\hline Total & 100.11 & 100.06 & 100.00 & 100.05 & 99.95 & 100.03 & 99.89 & 99.94 & 100.04 & 100.01 \\
\hline $\mathrm{CO}_{2}$ & 1.80 & 2.27 & 0.95 & & 0.93 & & & & & 7.00 \\
\hline Mg\# & 51.4 & 52.1 & 52.3 & 50.7 & 28.8 & 61.9 & 54.8 & 54.8 & 41.4 & 52.2 \\
\hline $\mathrm{Zn}$ & 84 & 87 & 87 & 115 & 124 & 63 & 79 & 97 & 115 & 63 \\
\hline $\mathrm{Cu}$ & 77 & 94 & 92 & 62 & 27 & 95 & 55 & 12 & 35 & 27 \\
\hline $\mathrm{Sc}$ & 42 & 39 & 44 & 55 & 48 & 26 & 19 & 40 & 28 & 32 \\
\hline $\mathrm{Ga}$ & 16 & 14 & 16 & 18 & 17 & 14 & 21 & 18 & 16 & 19 \\
\hline $\mathrm{Ni}$ & 139 & 139 & 139 & 106 & 69 & 40 & 32 & 15 & 140 & 213 \\
\hline $\mathrm{Co}$ & 39 & 41 & 41 & 33 & 32 & 33 & 26 & 24 & 35 & 36 \\
\hline $\mathrm{Cr}$ & 389 & 371 & 372 & 197 & 273 & 266 & 67 & 18 & 392 & 305 \\
\hline $\mathrm{V}$ & 390 & 377 & 370 & 300 & 368 & 194 & 176 & 380 & 317 & 333 \\
\hline $\mathrm{Ba}$ & 83 & 75 & 73 & 69 & 130 & 132 & 116 & 173 & 274 & 85 \\
\hline $\mathrm{Pb}$ & 4 & 3 & 3 & 5 & n.d. & 3 & 5 & 6 & 6 & 6 \\
\hline $\mathrm{Zr}$ & 74 & 73 & 73 & 97 & 98 & 75 & 84 & 88 & 135 & 84 \\
\hline & $(I C P-M S)$ & & $(I C P-M S)$ & $(I C P-M S)$ & & $(I C P-M S)$ & & $(I C P-M S)$ & $(I C P-M S)$ & \\
\hline $\mathrm{Rb}$ & 6.35 & 5.00 & 25.2 & 28.4 & 17.0 & 3.15 & 2.00 & 14.8 & 68.8 & 2.00 \\
\hline $\mathrm{Sr}$ & 130 & 136 & 129 & 385 & 201 & 429 & 120 & 129 & 160 & 96 \\
\hline $\mathrm{Y}$ & 31.8 & 27.0 & 14.8 & 19.9 & 40.0 & 12.5 & 29.0 & 26.3 & 28.1 & 26.0 \\
\hline $\mathrm{La}$ & 5.63 & 5.00 & 2.76 & 3.15 & 3.00 & 5.16 & 3.00 & 3.77 & 14.5 & 13.0 \\
\hline $\mathrm{Ce}$ & 14.0 & 12.0 & 7.84 & 9.59 & n.d. & 11.8 & 9.0 & 10.1 & 26.2 & 31.0 \\
\hline $\operatorname{Pr}$ & 2.18 & & 1.32 & 1.59 & & 1.63 & & 1.67 & 3.38 & \\
\hline $\mathrm{Nd}$ & 10.1 & 9.00 & 6.49 & 7.88 & 13.0 & 7.59 & 10.0 & 8.91 & 14.0 & 10.0 \\
\hline $\mathrm{Sm}$ & 3.24 & & 2.16 & 2.61 & & 2.20 & & 2.99 & 3.59 & \\
\hline $\mathrm{Eu}$ & 1.13 & & 0.778 & 0.907 & & 0.811 & & 1.10 & 1.06 & \\
\hline $\mathrm{Gd}$ & 4.21 & & 2.68 & 3.21 & & 2.72 & & 3.99 & 4.13 & \\
\hline $\mathrm{Tb}$ & 0.752 & & 0.470 & 0.560 & & 0.463 & & 0.714 & 0.698 & \\
\hline Dy & 5.04 & & 3.03 & 3.63 & & 2.95 & & 4.70 & 4.42 & \\
\hline Ho & 1.15 & & 0.638 & 0.791 & & 0.626 & & 1.01 & 0.869 & \\
\hline $\mathrm{Er}$ & 3.23 & & 1.70 & 2.15 & & 1.75 & & 2.78 & 2.43 & \\
\hline $\mathrm{Tm}$ & 0.478 & & 0.247 & 0.320 & & 0.258 & & 0.401 & 0.354 & \\
\hline $\mathrm{Yb}$ & 3.18 & & 1.52 & 2.03 & & 1.69 & & 2.54 & 2.24 & \\
\hline $\mathrm{Lu}$ & 0.492 & & 0.224 & 0.304 & & 0.258 & & 0.362 & 0.322 & \\
\hline $\mathrm{Nb}$ & 7.81 & 9.00 & 2.88 & 3.36 & 5.00 & 5.25 & 4.00 & 2.74 & 25.7 & 19.0 \\
\hline Hf & 2.78 & 3.00 & 1.61 & 2.24 & n.d. & 2.45 & 3.00 & 1.81 & 3.51 & n.d. \\
\hline $\mathrm{Ta}$ & 0.404 & & 0.161 & 0.188 & & 0.277 & & 0.159 & 1.480 & \\
\hline $\mathrm{Th}$ & 0.619 & n.d. & 0.112 & 0.211 & 1.00 & 0.342 & n.d. & 0.195 & 2.51 & 3.00 \\
\hline $\mathrm{U}$ & 0.151 & & 0.071 & 0.008 & & 0.102 & & 0.052 & 0.573 & \\
\hline$(\mathrm{La} / \mathrm{Sm})_{\mathrm{N}}$ & 1.12 & & 0.82 & 0.78 & & 1.51 & & 0.81 & 2.61 & \\
\hline$(\mathrm{Sm} / \mathrm{Yb})_{\mathrm{N}}$ & 1.13 & & 1.58 & 1.43 & & 1.45 & & 1.31 & 1.78 & \\
\hline$(\mathrm{La} / \mathrm{Yb})_{\mathrm{N}}$ & 1.27 & & 1.30 & 1.12 & & 2.19 & & 1.06 & 4.65 & \\
\hline $\mathrm{Ti} / \mathrm{V}$ & 25 & 25 & 25 & 31 & 30 & 40 & 53 & 25 & 38 & 31 \\
\hline $\mathrm{Nb} / \mathrm{Y}$ & 0.27 & 0.30 & 0.14 & 0.12 & 0.20 & 0.25 & 0.20 & 0.09 & 0.86 & 0.70 \\
\hline $\mathrm{Nb} / \mathrm{Yb}$ & 2.46 & & 1.90 & 1.66 & & 3.12 & & 1.08 & 11.46 & \\
\hline$(\mathrm{Th} / \mathrm{Ta}) /(\mathrm{Th} / \mathrm{Tb})$ & 1.86 & & 2.91 & 2.98 & & 1.67 & & 4.50 & 0.88 & \\
\hline
\end{tabular}

Abbreviations: bas - basalt; tra - trachyte; Fe-bas - ferrobasalt; trans - transitional-type; sub-alk - sub-alkaline-type; OIB - ocean-island basalt; P-MORB - plume-type mid-ocean ridge basalt; E-MORB - enriched-type mid-ocean ridge basalt; G-MORB - garnet-influenced mid-ocean ridge basalt; E - Early; $\mathrm{M}$ - Middle; L - Late; $\mathrm{Jr}$ - Jurassic; $\mathrm{Cr}$ - Cretaceous; mlf - massive lava flow; n.d. - not detected. $\mathrm{Mg}$ no. $=100 \times \mathrm{Mg} /\left(\mathrm{Mg}+\mathrm{Fe}^{2+}\right) . \mathrm{Fe}_{2} \mathrm{O}_{3}=0.15 \times \mathrm{FeO}$. Normalizing values for REE ratios are from Sun \& McDonough (1989). 


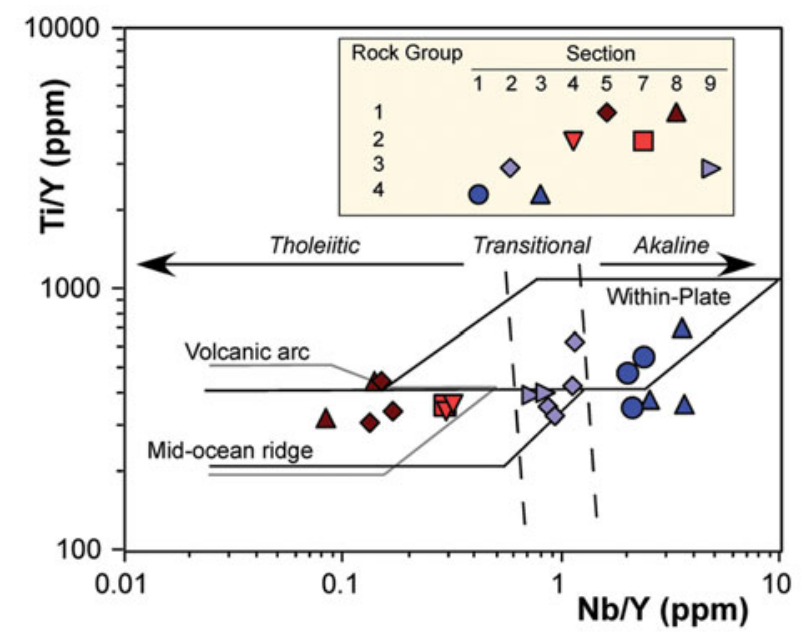

Figure 6. (Colour online) Ti/Y v. Nb/Y discrimination diagram (Pearce, 1982) for Middle Jurassic - Early Cretaceous volcanic rocks from the Ankara Mélange. Modified from Bortolotti et al. (2013a).

patible element patterns (Fig. 8a). The REE abundance (Fig. $8 \mathrm{~b}$ ) varies from $\sim 10$ to $\sim 20$ times that of chondrite and displays LREE/MREE smoothly depleted patterns with $(\mathrm{La} / \mathrm{Sm})_{\mathrm{N}}$ ratios ranging from 0.78 to 0.82 . The overall geochemical features of these rocks, as well as both the incompatible elements and REE patterns, resemble those of typical N-MORB (normal mid-ocean ridge basalt) (Sun \& McDonough, 1989). Nonetheless, HREEs are slightly depleted with respect to both LREEs and MREEs (Fig. 8b) with $(\mathrm{La} / \mathrm{Yb})_{\mathrm{N}}$ ratios $=1.06-1.30$ and $(\mathrm{Sm} / \mathrm{Yb})_{\mathrm{N}}$ ratios $=1.31-1.58$. In particular, the $(\mathrm{Sm} / \mathrm{Yb})_{\mathrm{N}}$ ratios are higher than that of typical N-MORB $\left(\mathrm{Sm}_{\mathrm{N}} / \mathrm{Yb}_{\mathrm{N}}=0.96\right.$, Sun \& McDonough, 1989). These values are comparable to those of garnet-influenced MORBs (G-MORB) from the External Ligurides of the Northern Apennines $\left(\mathrm{Sm}_{\mathrm{N}} / \mathrm{Yb}_{\mathrm{N}}\right.$ $=1.25-1.50$, Montanini, Tribuzio \& Vernia, 2008), Corsica $\left(\mathrm{Sm}_{\mathrm{N}} / \mathrm{Yb}_{\mathrm{N}}=1.30-1.81\right.$, Saccani et al. $\left.2008 b\right)$ and Elba Island $\left(\mathrm{Sm}_{\mathrm{N}} / \mathrm{Yb}_{\mathrm{N}}=1.49-2.10\right.$, Saccani \& Principi, 2016), as well as to those of the Palaeozoic Misho mafic complex in north Iran (Saccani et al. 2013a) and the Mesozoic Kermanshah ophiolites in south Iran (Saccani et al. 2013b). The G-MORB affinity of Group 1 rocks is also suggested by $\mathrm{Th}-\mathrm{Nb}$ co-variation and by their $(\mathrm{Ce} / \mathrm{Yb})_{\mathrm{N}}$ and $(\mathrm{Dy} / \mathrm{Yb})_{\mathrm{N}}$ ratios (Fig. 9), although in the most common tectonic discrimination diagrams these basalts plot in the field for N-MORBs (e.g. Fig. 10).

Group 2 is represented by basalts cropping out in Sections 4 and 7 . These rocks have a sub-alkaline nature with low $\mathrm{Nb} / \mathrm{Y}$ ratios ranging from 0.27 to 0.40 (Fig. 6) and show relatively high $\mathrm{TiO}_{2}, \mathrm{P}_{2} \mathrm{O}_{5}, \mathrm{Zr}$ and $\mathrm{Y}$ contents (Table 1). Ni, though variable, is generally low, whereas $\mathrm{Cr}$ and $\mathrm{V}$ are rather high. As exemplified in the variation diagrams in Figure 7, these rocks have major-element contents and compositions of many trace elements largely overlapping those of Group 1 basalts. Nonetheless, as a distinctive fea- ture, Group 2 basalts are characterized by large ion lithophile element (LILE)/high-field-strength element (HFSE) smoothly enriched patterns (Figs 8c). Likewise, REE patterns show a slight enrichment in LREEs compared to HREEs (Fig. 8d), with $(\mathrm{La} / \mathrm{Sm})_{\mathrm{N}}$ and $(\mathrm{La} / \mathrm{Yb})_{\mathrm{N}}$ ratios ranging from 1.12 to 1.51 and from 1.27 to 2.19 , respectively. These patterns are similar to that of the typical enriched-type MORB (E-MORB) of Sun \& McDonough (1989). The Th-Nb values are also similar to those of the typical E-MORB (Fig. 9a). In the most common tectonic discrimination diagrams these basalts plot across the boundary between the fields for N-MORB and E-MORB (e.g. Fig. 10).

Group 3 is represented by pillow basalts and ferrobasalts from Section 2, as well as by massive lava basalts from Section 9. These rocks have a transitional nature, as testified to by their high $\mathrm{Nb} / \mathrm{Y}$ ratios (Fig. 6). Basalt TU10.15 (Section 2) displays a rather primitive composition with relatively high $\mathrm{Mg}$ no. and low $\mathrm{TiO}_{2}$ and $\mathrm{P}_{2} \mathrm{O}_{5}$ contents (Table 1). In contrast, ferrobasalts from Section 2 and basalts from Section 9 have rather evolved compositions with relatively low $\mathrm{Mg}$ no. and very high $\mathrm{TiO}_{2}$ and $\mathrm{P}_{2} \mathrm{O}_{5}$ contents (Table 1). Accordingly, $\mathrm{Ni}$ and $\mathrm{Cr}$ contents are generally high in basalts, whereas they are relatively low in the differentiated rocks. Except for Mg no., the variation of many elements with respect to $\mathrm{Zr}$ display roughly common evolutionary trends towards high contents of the incompatible elements and $\mathrm{FeO}_{\mathrm{t}}$ and low contents of the compatible elements for all Group 3 samples (Fig. 7). In contrast, $\mathrm{Mg}$ no. for samples from Sections 2 and 9 show distinct evolutionary trends with respect to Zr. All these trends are compatible with a magmatic evolution by fractional crystallization. The $\mathrm{Th} / \mathrm{Tb}$ ratios of these rocks are significantly higher than those of Groups 1 and 2 rocks and significantly lower than those of Group 4 rocks (Fig. 7). Group 3 rocks show high abundance in low-field-strength elements (LFSEs) with respect to N-MORB and display regularly decreasing N-MORB normalized patterns from $\mathrm{Rb}$ to $\mathrm{Y}$ (Fig. 8e). The REE abundance (Fig. 8f) varies from $\sim 30$ to $\sim 100$ and from $\sim 10$ to $\sim 12$ times that of chondrite for LREEs and HREEs, respectively. These rocks displays LREE/MREE and LREE/HREE enriched patterns, with $(\mathrm{La} / \mathrm{Sm})_{\mathrm{N}}$ ratios $=2.61-4.15$ and $(\mathrm{La} / \mathrm{Yb})_{\mathrm{N}}$ ratios $=4.65-9.17$. These incompatible elements and REE patterns are comparable to those of P-MORB (plume-type MORB). This conclusion is also supported by the co-variation of the $\mathrm{Th}$ and $\mathrm{Nb}$ concentrations (Fig. 9a). In the most common tectonic discrimination diagrams (e.g. Fig. 10) these rocks generally plot in the fields for E-MORBs and alkaline ocean-island basalts (OIB).

Group 4 is represented mainly by alkaline basalts and subordinate trachytes from Sections 1 and 3. These rocks have a clear alkaline nature, as testified to by their very high $\mathrm{Nb} / \mathrm{Y}$ ratios (Fig. 6). Alkaline basalts from Section 3 mainly display relatively primitive compositions, whereas alkaline basalts from Section 1 represent rather evolved compositions. Generally, all 

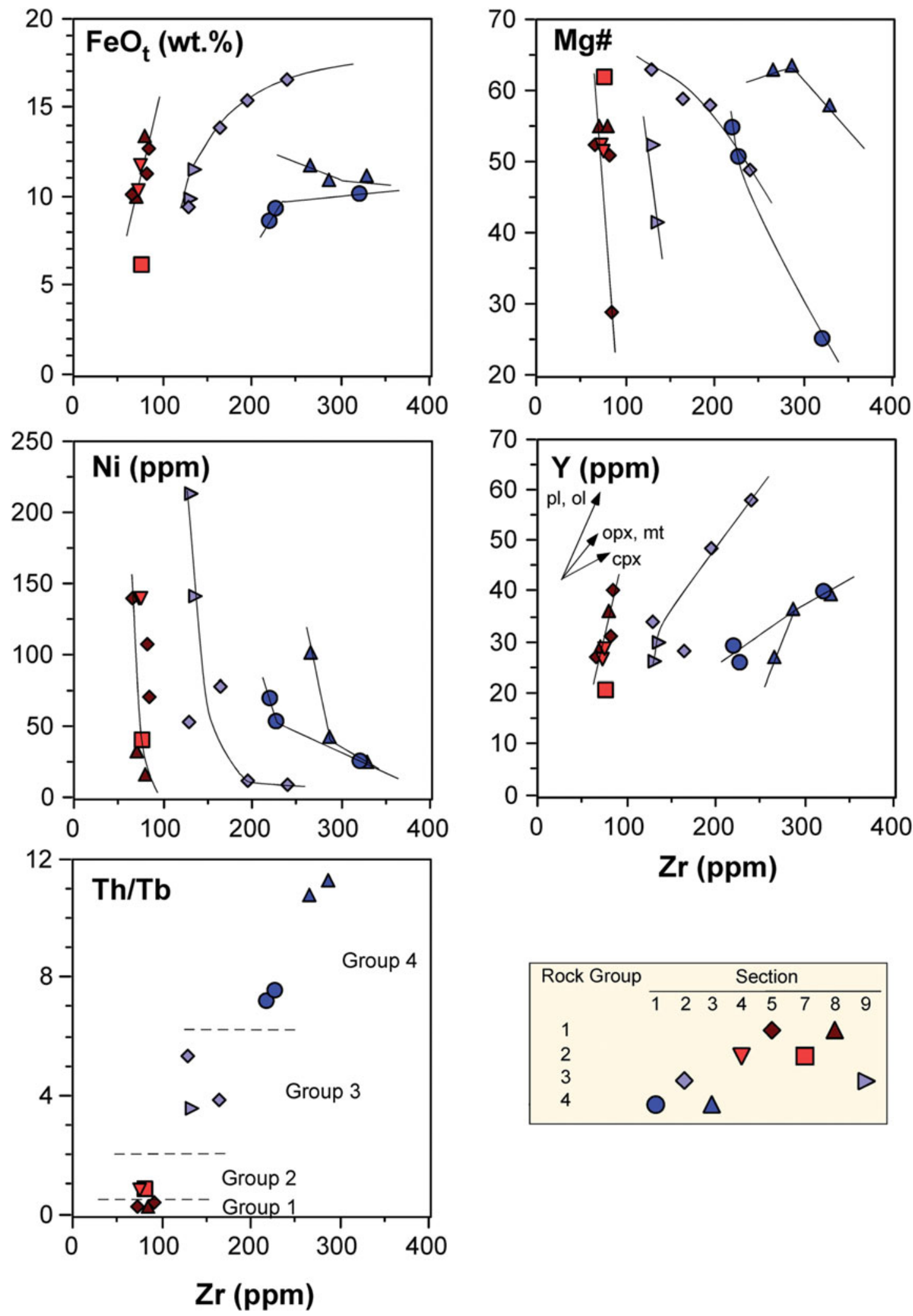

Figure 7. (Colour online) Variation diagrams for some representative major and trace elements versus Zr for Middle Jurassic - Early Cretaceous volcanic rocks from the Ankara Mélange. Major elements are recalculated on a volatile-free and calcite-free basis. Abbreviations: pl - plagioclase; ol - olivine; cpx - clinopyroxene; opx - orthopyroxene; mt - magnetite. $\mathrm{Mg} \mathrm{no.}=100 \times \mathrm{Mg} /\left(\mathrm{Mg}+\mathrm{Fe}{ }^{2+}\right)$. Lines represent the inferred fractionation trends for the different rock groups. 

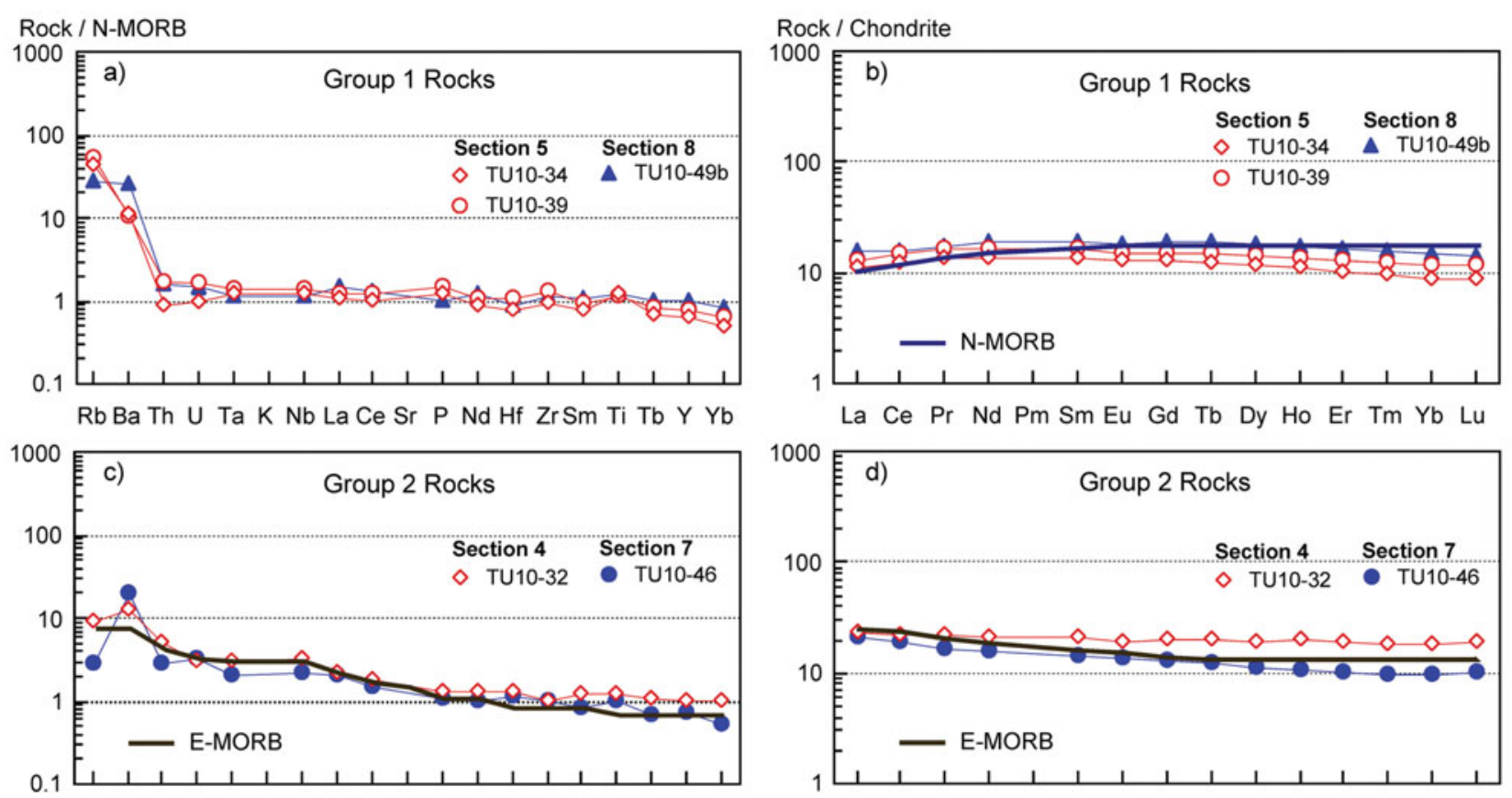

$\mathrm{Rb} B a \mathrm{Th} \mathrm{U}$ Ta K NbLaCe Sr P Nd Hf Zr Sm Ti Tb Y Yb

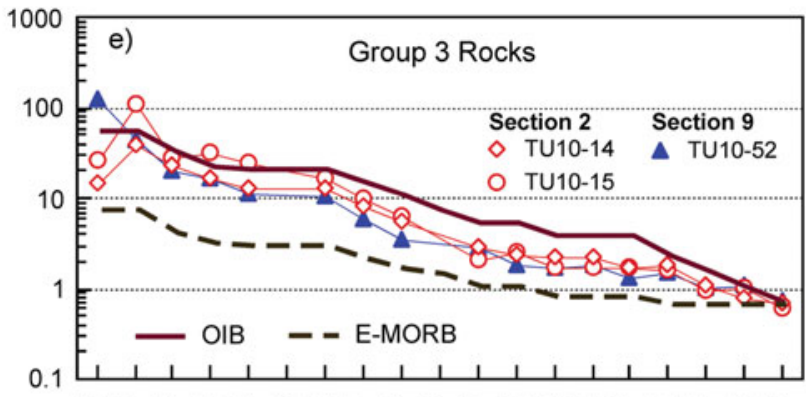

Rb Ba Th U Ta K NbLaCe Sr P Nd Hf Zr Sm Ti Tb Y Yb

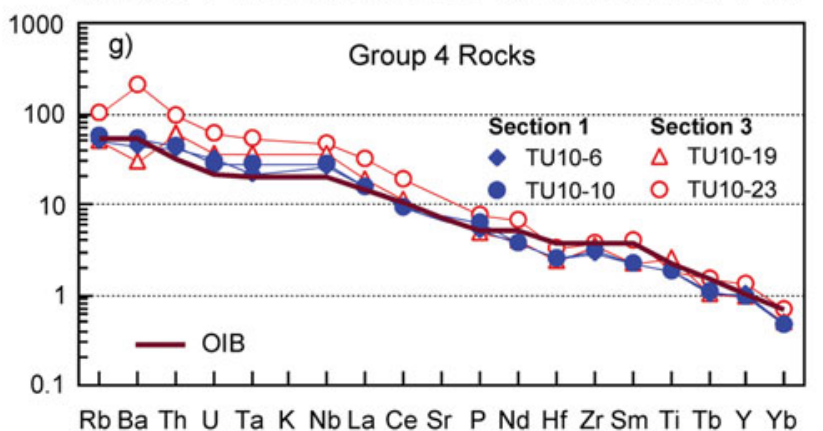

La Ce Pr Nd Pm Sm Eu Gd Tb Dy Ho Er Tm Yb Lu

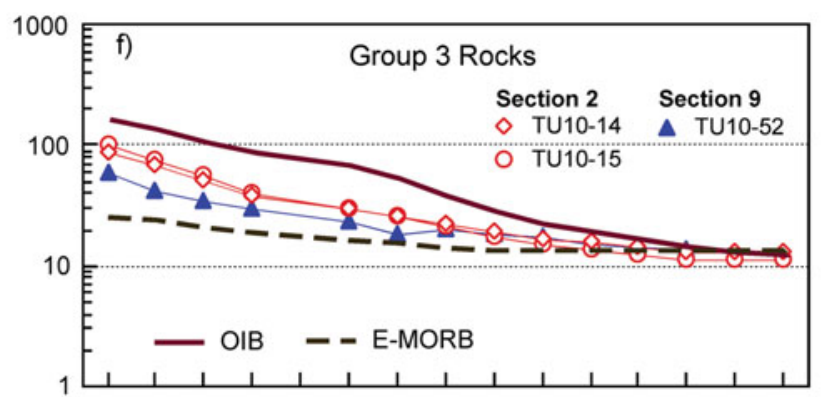

La Ce Pr Nd Pm Sm Eu Gd Tb Dy Ho Er Tm Yb Lu

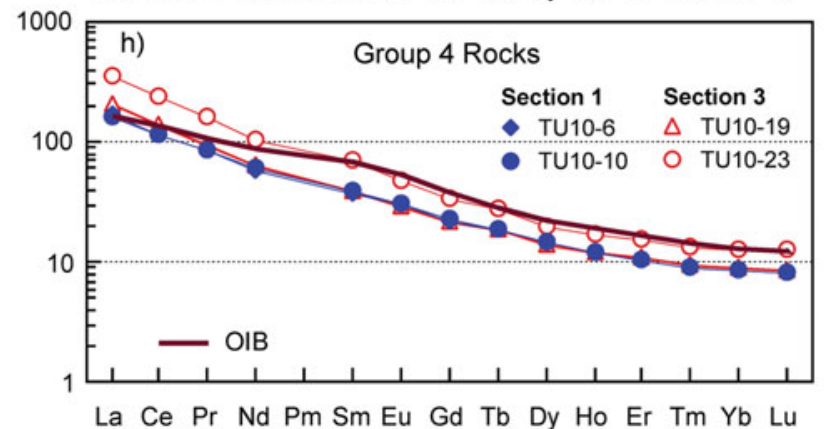

Figure 8. (Colour online) (a, c, e, g) N-MORB normalized incompatible element patterns and (b, d, f, h) chondrite-normalized REE patterns for Middle Jurassic - Early Cretaceous volcanic rocks from the Ankara Mélange. Normalizing values and the compositions of normal mid-ocean ridge basalt (N-MORB), enriched mid-ocean ridge basalt (E-MORB) and ocean-island basalt (OIB) are from Sun \& McDonough (1989).

samples have high contents of $\mathrm{TiO}_{2}, \mathrm{P}_{2} \mathrm{O}_{5}, \mathrm{Zr}, \mathrm{Nb}$, $\mathrm{Hf}$ and $\mathrm{Th}$ and low contents of compatible elements (Table 1; Fig. 7). In the variation diagrams in Figure 7, rocks from the different sections show different evolutionary trends, most likely reflecting the distinct evolutions of magmas of different initial compositions. This is particularly evident in the $\mathrm{FeO}_{\mathrm{t}}, \mathrm{Mg}$ no., $\mathrm{Ni}$ and $\mathrm{Y}$ v. Zr diagrams. Nonetheless, the well-defined trends observed for samples from each single section suggest that each section consists of rocks belonging to a comagmatic suite. Group 4 rocks show high abundance in LFSEs with respect to N-MORB and display regularly decreasing N-MORB normalized patterns from $\mathrm{Rb}$ to $\mathrm{Y}$ (Fig. 8g). The REE abundance (Fig. 8h) varies from $\sim 60$ to $\sim 400$ and from $\sim 9$ to $\sim 10$ times that of chondrite for LREEs and HREEs, respectively. These rocks displays LREE/MREE and LREE/HREE strongly enriched patterns, with $(\mathrm{La} / \mathrm{Sm})_{\mathrm{N}}$ ratios $=$ 4.15-5.25 and $(\mathrm{La} / \mathrm{Yb})_{\mathrm{N}}$ ratios $=18.75-23.13$. Group 4 rocks can readily be distinguished from Group 

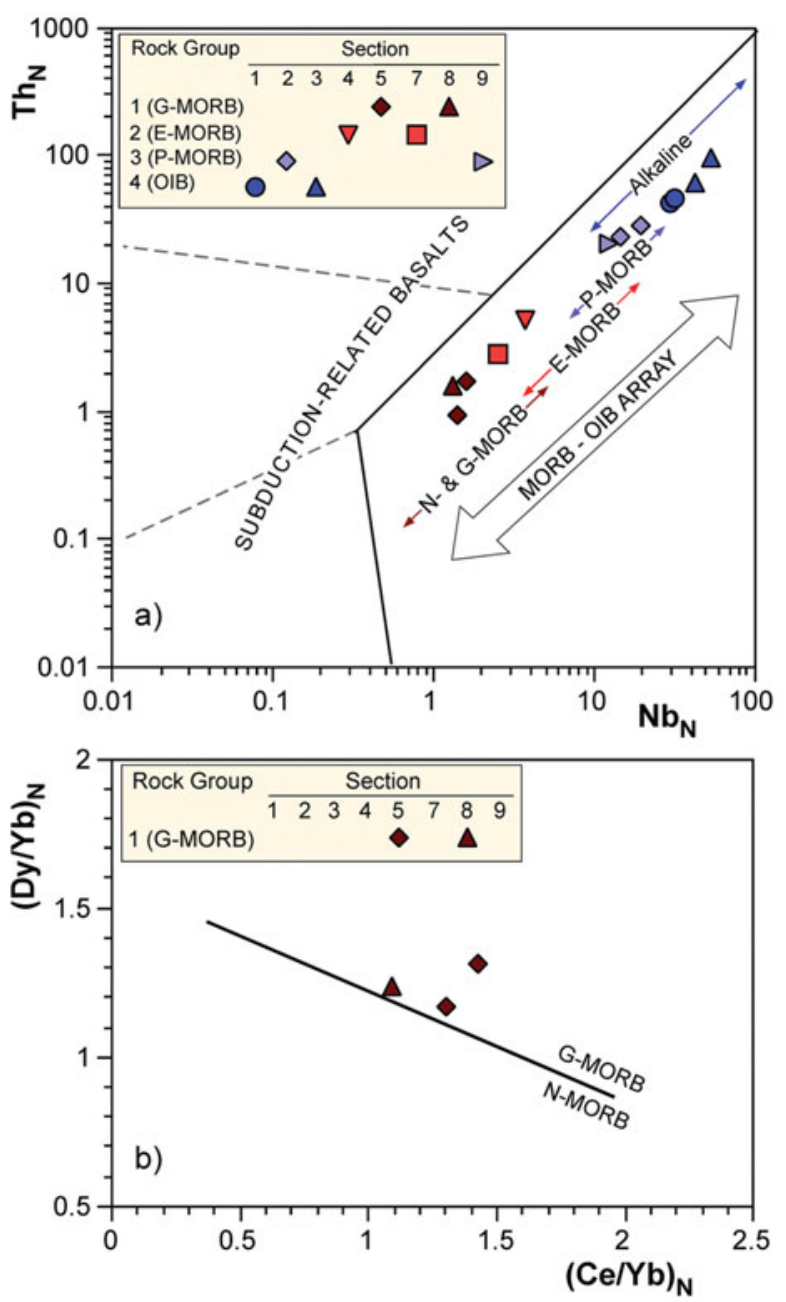

Figure 9. (Colour online) (a) $\mathrm{Th}_{\mathrm{N}} \mathrm{v} \cdot \mathrm{Nb}_{\mathrm{N}}$ and (b) $(\mathrm{Dy} / \mathrm{Yb})_{\mathrm{N}} \mathrm{v}$. $(\mathrm{Ce} / \mathrm{Yb})_{\mathrm{N}}$ discrimination diagrams for Middle Jurassic - Early Cretaceous volcanic rocks from the Ankara Mélange. Modified after Saccani (2015). N-MORB and chondrite normalization values for panels (a) and (b), respectively, are from Sun \& McDonough (1989).

3 rocks, as they show higher $\mathrm{Zr}$, incompatible element and LREE concentrations, as well as $\mathrm{Th} / \mathrm{Tb}$ ratios at comparable Mg no. (Figs 7, 8g, h). The overall geochemistry of these basalts resembles that of alkaline basalts generated at within-plate ocean-island settings (OIB), as also exemplified by the generally high Ti/V ratios (Table 1). This conclusion is also supported by the co-variation of the $\mathrm{Th}$ and $\mathrm{Nb}$ values (Fig. 9), as well as by the most common tectonic discrimination diagrams (e.g. Fig. 10).

\section{Discussion}

\section{6.a. Mantle melting processes and magma generation}

One of the main goals of this study is to assess the nature and tectonic significance of the magmatic events that occurred in the Turkish sector of the Neotethys during the Middle Jurassic - Early Cretaceous time span. According to many authors (e.g. Pearce, 1982), the compositional differences between

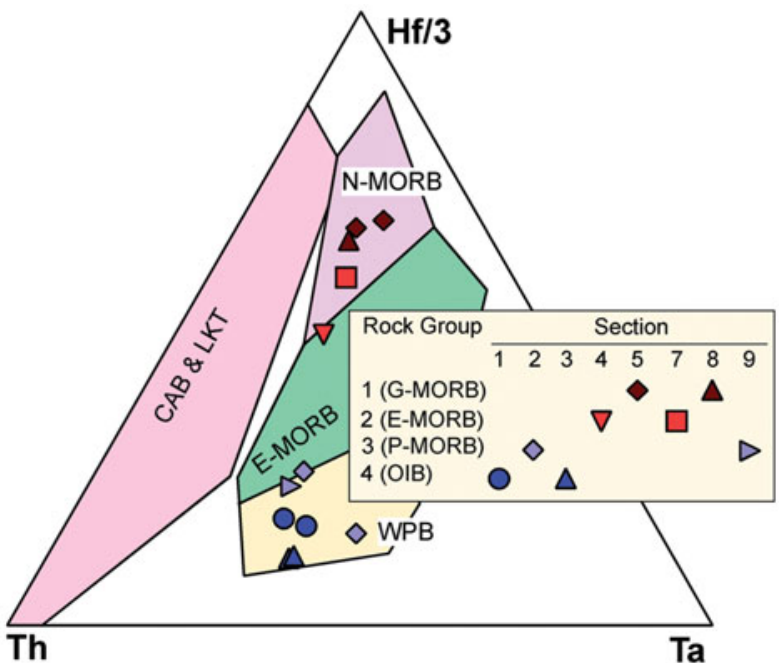

Figure 10. (Colour online) Th-Ta-Hf/3 (Wood, 1980) discrimination diagrams for Middle Jurassic - Early Cretaceous volcanic rocks from the Ankara Mélange.

magma types are related to different source characteristics that are associated, in turn, with distinct tectonomagmatic settings of formation. We will therefore focus our petrogenetic discussion on the identification of the possible mantle sources and related tectonic setting of formation of the four distinct lava groups identified in the previous section, which are: (1) G-MORBs (Group 1); (2) E-MORBs (Group 2); (2) P-MORBs (Group 3); (4) OIB-type alkaline volcanic rocks (Group 4). Unfortunately, the chemical variation due to fractional crystallization cannot be defined in detail, as the mélange nature of the sampled rocks prevents us from establishing definite genetic relationships between rocks within each single chemical group. Nonetheless, some trace-element ratios (e.g. $\mathrm{Zr} / \mathrm{Nb}, \mathrm{Ce} / \mathrm{Y}, \mathrm{Th} / \mathrm{Ta}, \mathrm{Th} / \mathrm{Tb}$ ) are little affected by fractional crystallization of predominantly olivine + clinopyroxene + plagioclase. Therefore, even in the presence of significant amounts of fractionation, they are believed to represent the elemental ratios in the source (e.g. Allègre \& Minster, 1978; Beker et al. 1997). Ratios of incompatible elements (Ce/Y, $\mathrm{Nb} / \mathrm{Yb}$ ), ratios of hygromagmatophile element ratios $((\mathrm{Th} / \mathrm{Ta}) /(\mathrm{Th} / \mathrm{Tb}))$ (Table 1$)$, as well as distinct normalized multi-element and REE patterns (Fig. 8) suggest that the different magmatic rocks from the Ankara Mélange units have been most likely originated from chemically distinct mantle sources. In order to constrain the possible mantle sources of the different rock series of the Ankara Mélange, non-modal, batch partial melting models are presented in Figure 11. A rigorous quantification of the melting processes is not possible as the composition of the mantle sources are difficult to constrain. However, a semi-quantitative modelling of the REEs can place some solid constraints. The melt modelling uses plots of LREE/HREE (i.e. La/Yb) v. MREE/HREE (i.e. Dy/Yb) ratios, which are particularly useful for distinguishing between melting in the 

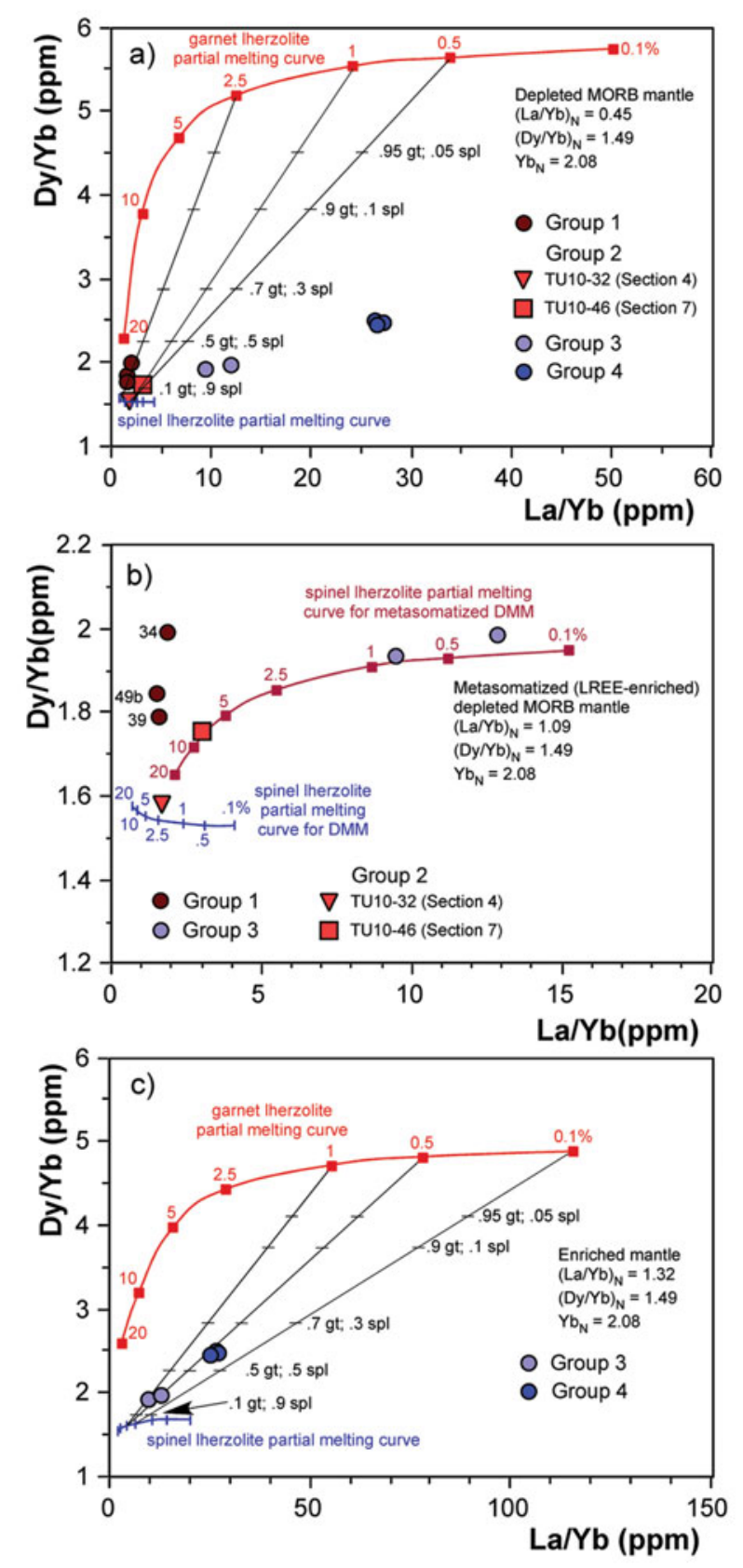

Figure 11. (Colour online) Melt curve models based on $\mathrm{Dy} / \mathrm{Yb}$ $\mathrm{v}$. La/Yb. Melt curves are calculated using non-modal, batch melts of garnet and spinel lherzolites. (a) Melt curves for DMM mantle (Workman \& Hart, 2005); (b) melt curves for a theoretical DMM mantle (Workman \& Hart, 2005) enriched in LREEs by OIB-type components; (c) melt curves for a theoretical enriched (OIB-type) mantle. Garnet lherzolite mode is: $0.598 \mathrm{ol}$, $0.211 \mathrm{opx} ; 0.076 \mathrm{cpx}, 0.115 \mathrm{gt}$ that melts in the proportions 0.05 ol, 0.20 opx, $0.30 \mathrm{cpx}, 0.45$ gt. Spinel lherzolite mode is: 0.578 ol, $0.270 \mathrm{opx}, 0.119 \mathrm{cpx}, 0.033 \mathrm{spl}$ that melts in the proportions $0.10 \mathrm{ol}, 0.27 \mathrm{opx}, 0.50 \mathrm{cpx}, 0.13 \mathrm{spl}$. Mantle mode and melting proportions are from Thirlwall, Upton \& Jenkins (1994). Arrays representing the mixing between various proportions of melt fractions from the garnet-facies mantle and melt fractions from the spinel-facies mantle are also shown. Distribution coefficients are from Irving \& Frey (1984) with the exception of those for spinel, which are from McKenzie \& O'Nions (1991). Normalizing values are from Sun \& McDonough (1989). spinel and garnet stability fields (Thirlwall, Upton \& Jenkins, 1994). Partial melting of a mantle source in the spinel-facies produces little change in $\mathrm{Dy} / \mathrm{Yb}$ ratios in melts with respect to melt fraction. In contrast, mantle partial melting in the garnet-facies produces large changes in $\mathrm{Dy} / \mathrm{Yb}$ ratios with melt fraction. In both cases, $\mathrm{La} / \mathrm{Yb}$ ratios are particularly responsive to melt fraction change (Fig. 11). Another important feature of these plots is that mixing between different melt fractions will generate linear mixing arrays (e.g. Beker et al. 1997).

Group 1 basalts. As observed in the previous section, the Group 1 rocks (G-MORBs) show unusually high MREE/HREE ratios $\left(\mathrm{Dy}_{\mathrm{N}} / \mathrm{Yb}_{\mathrm{N}}=1.20\right.$ 1.34) compared to the typical N-MORB $\left(\mathrm{Dy}_{\mathrm{N}} / \mathrm{Yb}_{\mathrm{N}}\right.$ $=1$, Sun \& McDonough, 1989). REE modelling for Group 1 basalts (Fig. 11a) shows that these rocks cannot have been simply derived from partial melting of a typical depleted MORB mantle (DMM) source (Workman \& Hart, 2005) in the spinel-facies. Rather, their significant HREE/MREE fractionation can be interpreted as a garnet signature, which can be related either to a deep initiation of melting in the garnet peridotite stability field, or to the melting of a heterogeneous mantle source characterized by garnet-bearing mafic/ultramafic layers (e.g. Montanini, Tribuzio \& Vernia, 2008; Saccani et al. 2008b; Saccani, 2015). However, melting of a DMM source bearing garnet mafic/ultramafic layers would generate primary melts characterized by high MREE/HREE ratios coupled with low $(<0.8)$ LREE/HREE ratios (not shown). By consequence, the high (1.06-1.30) LREE/HREE ratios observed in the Group 1 basalts are inconsistent with this hypothesis. Therefore, in Figure 11a the partial melting model of a DMM source that starts in the garnet-facies and continues to larger degrees in the spinel-facies (with various combinations of melting fractions in the garnet- and spinel-facies) is shown. It can be observed that the REE composition of the Group 1 basalts is compatible with the calculated compositions for $2.5 \%$ melting in the garnet-facies and $10 \%$ melting in the spinel-facies, assuming mixing of $\sim 70-80 \%$ of melt derived from spinel-facies mantle with $\sim 30-20 \%$ melt from garnetfacies mantle.

Group 2 basalts. Group 2 basalts (E-MORBs) show variable LREE/HREE enrichments (Fig. 8d), which can be considered a result of variable mixing between depleted and enriched asthenospheric sources or, alternatively, from lower degrees of partial melting of a DMM source, compared to N-MORBs. In fact, basalt TU10.32 is generally compatible with a low degree $(\sim 2.5 \%)$ of partial melting of a DMM source in the spinel-facies (Fig. 11b). However, basalt TU10.46 shows HREE values lower than those of the N-MORBs of Group 1, which cannot be generated by lower degrees of partial melting of a common mantle source (Fig. 8b, d). In addition, lower degrees of partial melting of a DMM source in the spinel-facies cannot generate the $(\mathrm{La} / \mathrm{Yb})_{\mathrm{N}}$ and $(\mathrm{Dy} / \mathrm{Yb})_{\mathrm{N}}$ ratios of this 
sample. The most appropriate solution for the genesis of this E-MORB sample is not straightforward. The LREE/HREE and MREE/HREE ratios are compatible with mixing of melt derived from a very low degree $(\sim 1 \%)$ of partial melting of a DMM source in the garnet-facies with melt derived from a much larger degree $(\sim 10 \%)$ of partial melting in the spinel-facies mantle (Fig. 11a). The REE composition of these basalts is compatible with mixing of $<10 \%$ of melts generated in the garnet-facies with $>90 \%$ of melts generated in the spinel-facies. Nonetheless, a possible alternative solution is to invoke a more enriched source than DMM. Figure $11 \mathrm{~b}$ shows that the melting curve of a hypothetical DMM source slightly enriched in LREEs with $(\mathrm{La} / \mathrm{Yb})_{\mathrm{N}}=1.09$ and $(\mathrm{Dy} / \mathrm{Yb})_{\mathrm{N}}=1.49$, and has a $\mathrm{Yb}$ concentration equal to that of DMM $\left(\mathrm{Yb}_{\mathrm{N}}=2.08\right)$. The model shows that the REE composition of E-MORB TU10.46 is compatible with the calculated composition for $\sim 8 \%$ partial melting of this theoretical source in the spinel-facies.

Group 3 and Group 4 rocks. The high LREE/HREE ratios displayed by the transitional basalts of Group 3 rocks (P-MORBs) and by the alkaline basalts of Group 4 (Fig. 8f, h) suggest an involvement of a garnet peridotite source. Moreover, the high $\mathrm{La} / \mathrm{Yb}$ ratio observed in these basalts implies a source more enriched in LREEs than DMM. In fact, variable degrees of partial melting of a DMM source in the spinel-facies cannot generate the observed $\mathrm{La} / \mathrm{Yb}$ ratios, and variable degrees of partial melting of a DMM source in the garnet-facies cannot generate the observed $\mathrm{La} / \mathrm{Yb}$ and $\mathrm{Dy} / \mathrm{Yb}$ ratios (Fig. 11a). Therefore, the most appropriate solution is to invoke a more enriched source than $\mathrm{DMM}$, although it is impossible to assess the exact composition of this source. The model in Figure 11c illustrates melting curves for a hypothetical LREEenriched source $\left(\right.$ Beker et al. 1997) with $(\mathrm{La} / \mathrm{Yb})_{\mathrm{N}}=$ 1.32 and $(\mathrm{Dy} / \mathrm{Yb})_{\mathrm{N}}=1.49$, and has a $\mathrm{Yb}$ concentration equal to that of DMM $\left(\mathrm{Yb}_{\mathrm{N}}=2.08\right)$. Higher $\mathrm{Yb}$ concentrations (e.g. PM values) in the source would generate concentrations of HREEs in the melts that are too high compared with the Group 3 and Group 4 volcanic rocks.

The co-variation in $\mathrm{La} / \mathrm{Yb}-\mathrm{Dy} / \mathrm{Yb}$ systematics of Group 3 and Group 4 samples (Fig. 11c) cannot, however, be explained by variable degrees of partial melting of this enriched source in either the spinel- or garnet-facies. Melting in the garnet-facies produces melts with much higher $\mathrm{Dy} / \mathrm{Yb}$ ratios than those of both Group 3 and Group 4 samples at reasonable degrees of melting (i.e. $<20 \%$ ). Alternatively, the mantle source would require an unusually low $\mathrm{Dy} / \mathrm{Yb}$ ratio if the samples were to be simply the product of garnet-facies mantle melting. By contrast, melting in the spinel-facies produces melts with both $\mathrm{La} / \mathrm{Yb}$ and $\mathrm{Dy} / \mathrm{Yb}$ ratios lower than those of both Group 3 and Group 4 samples. Therefore, the simplest model to account for the REE systematics of these rocks involves mixing of small melt fractions from garnet-facies enriched mantle with relatively larger melt fractions from spinel-facies (Fig. 11c). This figure shows that the $\mathrm{La} / \mathrm{Yb}-\mathrm{Dy} / \mathrm{Yb}$ systematics of Group 3 basalts can be explained by mixing of small-degree melts $(\sim 0.5-$ $1 \%)$ and larger degree melts $(\sim 5 \%)$ from garnetand spinel-facies mantle, respectively. Likewise, the observed REE data for Group 4 basalts can be accounted for by mixing of melts from $\sim 0.5 \%$ and $\sim 5 \%$ partial melting from garnet- and spinel-facies mantle, respectively. Alternatively, the $\mathrm{La} / \mathrm{Yb}-\mathrm{Dy} / \mathrm{Yb}$ systematics of Group 4 basalts can be explained by a comparatively higher degree of melting $(\sim 1 \%)$ in the garnet-facies mantle and comparatively lower degree of melting $(\sim 2.5 \%)$ in the spinel-facies mantle. In any case, the different $\mathrm{La} / \mathrm{Yb}-\mathrm{Dy} / \mathrm{Yb}$ ratios shown by Group 3 and Group 4 basalts can be accounted for by mixing of different proportions of melts generated in the garnet- and spinel-facies mantle. In detail, Group 4 basalts may have resulted from the mixing of $\sim 60 \%$ of melt derived from spinel-facies mantle with $\sim 40 \%$ melt from garnet-facies mantle, whereas Group 3 basalts may have resulted from the mixing of $\sim 90 \%$ of melt derived from spinel-facies mantle with $\sim 10 \%$ melt from garnet-facies mantle (Fig. 11c).

Figure $11 \mathrm{~b}$ shows that the $\mathrm{La} / \mathrm{Yb}$ and $\mathrm{Dy} / \mathrm{Yb}$ ratios of Group 3 basalts can also be compatible with a very low degree $(<0.8 \%)$ of partial melting in the spinelfacies of the slightly metasomatized mantle source hypothesized for the genesis of Group 2 rocks. Such a very low degree of partial melting is, however, unreasonable. In fact, experimental studies on melt mobility in peridotites showed that very small melt fractions are not readily mobile and therefore they remain within the host peridotite rather than migrating to form volcanic rock melt (see Warren, 2016 for a more detailed discussion). Finally, it should be noted that variations in the degree of partial melting in the spinel field are difficult to constrain owing to the small range in $\mathrm{La} / \mathrm{Yb}$ ratios generated by spinel-facies melting. Some scatter in the $\mathrm{La} / \mathrm{Yb}-\mathrm{Dy} / \mathrm{Yb}$ systematics (Fig. 11c) might be accounted for by small fluctuations in the degree of melting of garnet-facies mantle. In any case, from a semi-quantitative point of view, melt fractions in the garnet field are restricted to $<1 \%$ whereas those in the spinel field are probably several per cent $(\sim 5 \%)$.

The possible influence of crustal contamination can be excluded as the $\mathrm{Th}-\mathrm{Nb}$ compositions of all rock types plot within the MORB-OIB array (Fig. 9). Other geochemical indicators further support this conclusion. For example, high $\mathrm{Th} / \mathrm{Ta}$ and low $\mathrm{Nb} / \mathrm{U}$ ratios are effective indicators of crustal contamination. All groups of Middle Jurassic - Early Cretaceous basalts from the Ankara Mélange show very low $\mathrm{Th} / \mathrm{Ta}$ ratios $(<1.74)$, as well as $\mathrm{Nb} / \mathrm{U}$ ratios averaging 44 in N-MORBs, 50 in E-MORBs, 40 in P-MORBs and 43 in OIBs. These $\mathrm{Nb} / \mathrm{U}$ ratios are comparable to those of the typical N-MORB (49.6), E-MORB (46.1) and OIB (47.1) of Sun \& McDonough (1989).

Bortolotti et al. (2013a) have noted that the association of depleted basalts (N-MORBs), moderately 


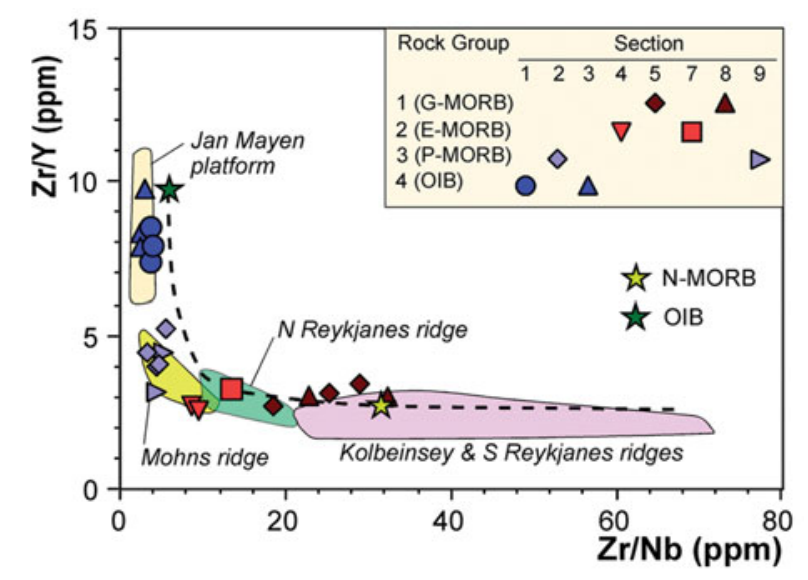

Figure 12. (Colour online) $\mathrm{Zr} / \mathrm{Y}$ v. $\mathrm{Zr} / \mathrm{Nb}$ diagram for volcanic rocks from the Middle Jurassic - Early Cretaceous volcanic rocks from the Izmir-Ankara Mélange (modified from Bortolotti et al. 2013a). The compositions of modern normal mid-ocean ridge basalt (N-MORB) and ocean-island basalt (OIB) are from Sun \& McDonough (1989). The compositional variation for ocean-floor basalts erupted in the North Atlantic Ocean is shown for comparison (data from Hanan et al. 2000). The dashed line represents the mixing curve calculated using the OIB and N-MORB end-members.

enriched basalts (E-MORBs) and variably enriched rocks (P-MORBs and OIBs) occurring in the Ankara Mélange is also observed in many peri-Mediterranean ophiolitic complexes (e.g. Saccani \& Photiades, 2005; Saccani et al. 2011 and references therein), as well as from several Middle East ophiolites (Allahyari et al. 2010; Saccani et al. 2010, 2013a,b), where it is interpreted as the result of partial melting of a MORBtype asthenospheric source enriched in HFSEs and LREEs by an OIB-type chemical component (plumetype component). Bortolotti et al. (2013a) have used the co-variation of $\mathrm{Zr} / \mathrm{Y}$ and $\mathrm{Zr} / \mathrm{Nb}$ to qualitatively depict the influence of a plume-type component on MORB compositions in the Middle Jurassic - Early Cretaceous basalts from the Ankara Mélange (Fig. 12). From Figure 12 it is evident that the data conform extremely well to the mixing curve calculated using the OIB and N-MORB end-members. Such mixing relationships are consistent with either magma mixing or source region mixing (or eventually, a combination of these).

\section{6.b. Tectonomagmatic significance}

The melting models carried out for the different groups of volcanic rocks, which are presented in the previous section, allow the following conclusions to be drawn: (1) the geochemically distinct groups of Middle Jurassic - Early Cretaceous volcanic rocks in the Ankara Mélange are related to different mantle source compositions and partial melting degrees; (2) regardless of their geochemical affinities, all the studied volcanic rocks were generated by partial melting starting in the garnet-facies mantle and continuing to larger degrees in the spinel-facies mantle. Perhaps, Group 2 basalts from Section 7 may represent the only ex- ception to this conclusion. In fact, their chemistry is compatible either with partial melting starting in the garnet-facies mantle and continuing to larger degrees in the spinel-facies mantle, or partial melting in the spinel-facies from a slightly enriched source. As shown before, the formation of enriched alkaline and P-MORB-type rocks implies the occurrence of mantle sources strongly metasomatized by OIB-type components. Two alternative hypotheses can account for such OIB-type metasomatism of depleted mantle sources: (1) the existence of plume activity in the region during Middle Jurassic - Early Cretaceous times and (2) the existence of deep mantle heterogeneously modified by previous mantle plume activity that occurred in the same area in association with the opening of the Neotethys. However, the extant geological evidence suggests that the first hypothesis can be disregarded. In fact, the lack of magmatic evolution from more depleted to more enriched rocks that is commonly observed in plume-related magmatism, the absence of basaltic plateaus and a relatively small volume of plume-related volcanic rocks collectively argue against the existence of a well-established, long-lasting mantle plume in the region. Therefore, we favour the hypothesis that the different Middle Jurassic - Early Cretaceous volcanic rock types from the Ankara Mélange were formed from partial melting of a strongly heterogeneous mantle, with OIB-type components inherited from a previous mantle plume activity associated with the opening of the Neotethys. In fact, the Anisian alkaline volcanic rocks intruding the Kütahya-Bolkardag have been interpreted as the early products of the rifting of the Neotethys Ocean with the involvement of a plume (Göncüoglu, Turhan \& Tekin, 2003; Göncüoglu, 2010; Akal et al. 2012). Recent studies on modern oceanic basins further support our favoured hypothesis. In fact, these studies have demonstrated that the upper mantle is much more heterogeneous than previously thought (e.g. Brunelli et al. 2006; Warren, 2016). The mantle heterogeneities can be either the result of earlier tectonic events or ancient episodes of melting, melt extraction and melt entrapment, and they can occur at a local or at a regional scale (e.g. Liu et al. 2008; Warren et al. 2009).

A possible tectonomagmatic model that can explain the formation of the different volcanic rocks from the Ankara Mélange during Middle Jurassic - Early Cretaceous times is shown in Figure 13. In this model, the OIB-type metasomatized portions are likely to be prominent in the asthenospheric mantle. The uprising asthenospheric mantle underwent polybaric partial melting, which started in the garnet-facies and continued in the spinel-facies. The strongly enriched alkaline and P-MORB rocks were generated from various, but limited degrees of polybaric partial melting of OIB-type metasomatized portions. G-MORB and E-MORB were generated from various degrees of polybaric partial melting of depleted asthenosphere portions. Alternatively, E-MORB (particularly, that of Section 7) may have been generated from partial 

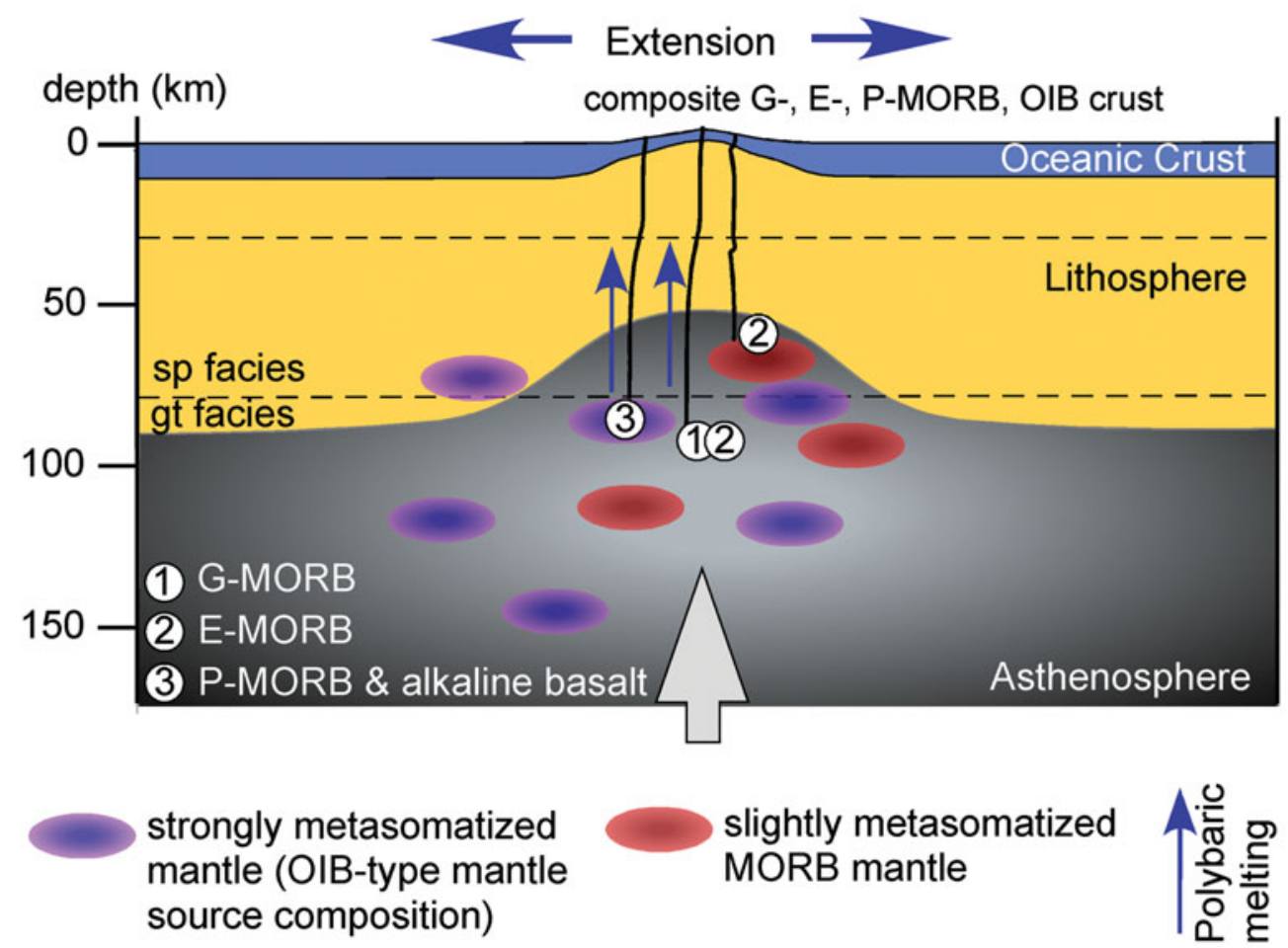

Figure 13. (Colour online) Two-dimensional cartoon showing the tectonomagmatic mechanisms responsible for the formation of garnet-influenced (G-), enriched (E-) and plume-type (P-) mid-ocean ridge basalts (MORB), as well as alkaline ocean-island-type (OIB) basalts from the Ankara Mélange during Late Jurassic - Early Cretaceous times. Other abbreviations: sp - spinel; gt - garnet.

melting of a slightly enriched mantle source in the spinel stability field. The tectonomagmatic model presented in Figure 13 implies that the different rocks were formed by partial melting of chemically different portions of the sub-oceanic mantle in different times. In consequence, this model does not necessarily imply the existence of a genetic relationship between different rock groups or within a single rock group.

\section{6.c. Geodynamic implications}

The ages obtained for the studied radiolarian assemblages coupled with the geochemistry of the associated basalts indicate that a composite oceanic crust including G-MORB, E-MORB, P-MORB and alkaline basalts was forming during Middle Jurassic - Early Cretaceous times. These ages are in agreement with the radiolarians (Bragin \& Tekin, 1996; Tekin, 1999; S. Celik, unpub. M.Sc. thesis, Hacettepe Univ., 2010; T. Üner, unpub. Ph.D. thesis, Hacettepe Univ., 2010; Tekin, Göncüoglu \& Uzuncimen, 2012; Göncüoglu et al. 2015) and foraminifera (Boccaletti, Bortolotti \& Sagri, 1966; Bortolotti \& Sagri, 1968; Yaliniz, Göncüoglu \& Özkan-Altiner, 2000; Rojay, Yaliniz \& Altiner, 2001) data obtained from different parts of the IAESB. The age ranges from the published biostratigraphical data indicate gaps in Early Jurassic and late Early Cretaceous times (see Göncüoglu, Sayit \& Tekin, 2010). Moreover, Middle Jurassic findings were restricted to a limited number of samples. Our new find- ings partially fill the gap during the late Early Cretaceous period (see Göncüoglu, Sayit \& Tekin, 2010). In fact, the radiolarian cherts associated with the GMORB of Section 8 indicated a late Valanginian early Barremian age.

The Middle Jurassic - Early Cretaceous rock assemblage found in the mélange complexes of the IAESB, as well as the tectonomagmatic model presented in the previous section can be framed within the geodynamic model presented by Göncüoglu (2010) (Fig. 14). According to this model, the rifting of the Neotethys Ocean started in Late Triassic time with the involvement of a mantle plume (Fig. 14a). Anisian and Norian volcanic rocks showing alkaline affinity were erupted at this stage (Göncüoglu, Turhan \& Tekin, 2003; Göncüoglu 2010; Akal et al. 2012; Bortolotti et al. 2013a). The Early Jurassic history of this oceanic basin cannot be straightforwardly constrained owing to the lack of data. In fact, Early Jurassic (HettanginianSinemurian) radiolarian chert blocks were found as single blocks in the Ankara Mélange, but they are not associated with volcanic rocks (S. Celik, unpub. M.Sc. thesis, Hacettepe Univ., 2010; Göncüoglu et al. 2015). Nonetheless, it is reasonable to postulate that during this time the Neotethys Ocean experienced an oceanic spreading phase. During the Middle Jurassic Early Cretaceous time span the formation of a composite oceanic crust including G-MORB, E-MORB, $\mathrm{P}-\mathrm{MORB}$ and alkaline basalts suggests that the volcanic rocks of the IAESB formed at a mid-ocean 
a) LATE TRIASSIC

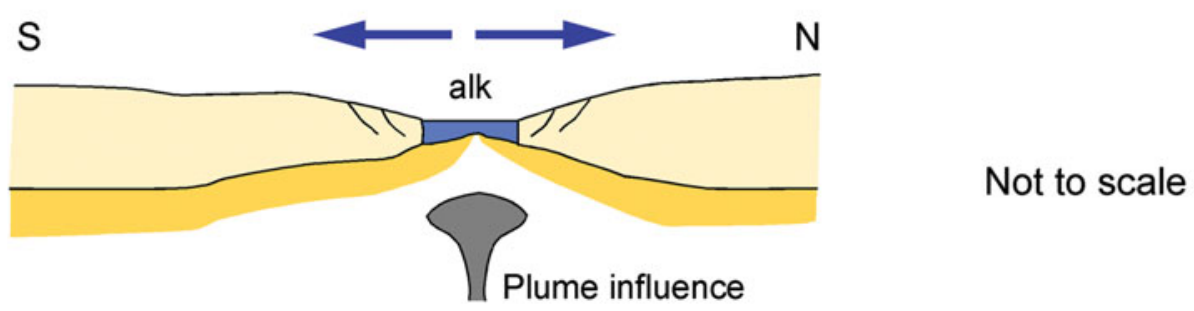

b) MIDDLE JURASSIC-EARLY CRETACEOUS

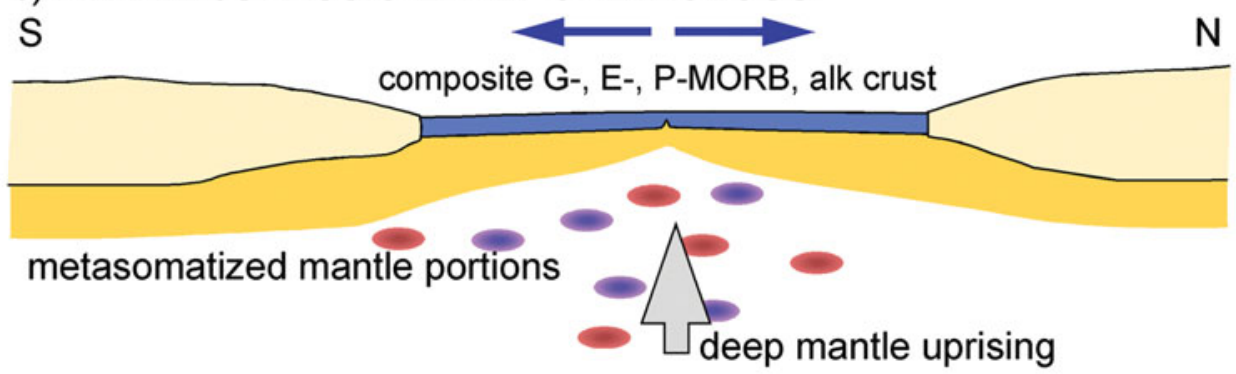

c) LATE EARLY CRETACEOUS

$S$

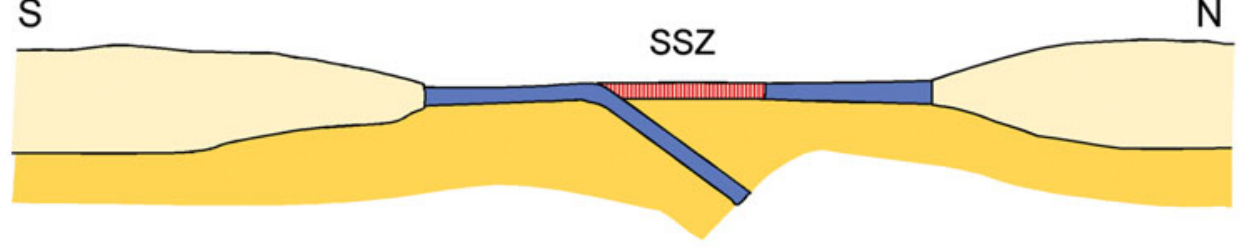

Figure 14. (Colour online) Two-dimensional cartoon showing the geodynamic evolution of the Izmir-Ankara Neotethys branch from Late Triassic to late Early Cretaceous times (modified from Göncüoglu, 2010). Abbreviations: MORB - mid-ocean ridge basalts; G- - garnet-influenced MORB; E- - enriched-MORB; P- - plume-type MORB; alk - alkaline basalts; SSZ - supra-subduction zone.

ridge setting by tapping different portions of a deep, highly heterogeneous mantle (Fig. 14b). Mantle heterogeneities consisted of portions variably enriched by OIB-type components, which were inherited from the Triassic mantle plume activity associated with the opening of this Neotethys oceanic branch (Fig. 14a). Our findings show that P-MORBs have a Middle Jurassic age, G-MORBs have a Late Jurassic age, EMORBs were erupted during Early Cretaceous time and alkaline basalts were erupted from Late Jurassic to Early Cretaceous times. Partial melting of different portions of a heterogeneous sub-oceanic mantle (Figs 13, 14b) can explain the formation of different and genetically unrelated magmatic rocks in the same time span.

The intra-oceanic subduction likely started from late Early Cretaceous time on the northern side of the Neotethys branch, leading to the production of suprasubduction zone (SSZ)-type rocks during late Early Cretaceous time (Fig. 14c). Previous data on the earliest ages related to supra-subduction type volcanism and the formation of a metamorphic sole indicate an early Late Cretaceous age (e.g. T. Üner, unpub. Ph.D. thesis, Hacettepe Univ., 2010); therefore, the intraoceanic decoupling within the ocean was considered as pre-Late Cretaceous in age. This age could not be veri- fied in this study, since in contrast to the western part of the IAESB, none of the basalt samples collected from the eastern part yielded supra-subduction characteristics. The different rock types formed in the ocean were then incorporated into the accretionary prism during the Late Cretaceous closure.

\section{Conclusion}

This study is focused on slide blocks including oceanic lavas associated with pelagic sediments within the eastern part of the Ankara Mélange. A detailed petrological characterization of the volcanic rocks and a detailed biochronological investigation of the associated radiolarites was carried out. The main conclusions can be summarized as follows.

(1) The radiolarian cherts associated with volcanic rocks show the following ages: late Valanginian to late Hauterivian (Section 1, OIB); earlymiddle Bajocian to late Bathonian-early Callovian (Section 2, P-MORB); middle-late Oxfordian to late Kimmeridgian-early Tithonian (Section 3, OIB); middle late Barremian-early early Aptian (Section 4, E-MORB); middle late Oxfordian to late Kimmeridgian-early Tithonian and early-early late Tithonian (Section 5, G-MORB); Valanginian to 
middle Aptian-early Albian (Section 7, E-MORB); late Valanginian-early Barremian (Section 8, GMORB).

(2) Volcanic rocks are largely represented by basalts and minor ferrobasalts and trachytes. They show different geochemical affinities and overlapping ages including: (a) Late Jurassic - Early Cretaceous GMORB; (b) Early Cretaceous E-MORB; (c) Middle Jurassic P-MORB; (d) Late Jurassic - Early Cretaceous alkaline basalts. All rock types show a clear garnet signature, as testified to by their high MREE/HREE ratios.

(3) REE modelling shows that their garnet signature is related to polybaric partial melting starting in the garnet peridotite stability field and continuing to larger degrees in the spinel-facies mantle. However, the different geochemical affinities displayed by the studied rocks are related to different mantle source compositions. G-MORBs were generated from $2.5 \%$ melting in the garnet-facies and $10 \%$ melting in the spinel-facies of a DMM source. E-MORB composition is compatible with mixing of melts derived from a very low degree $(\sim 1 \%)$ of partial melting of a DMM source in the garnet-facies with melts derived from a much larger degree $(\sim 10 \%)$ of partial melting in the spinel-facies mantle. Alternatively, these rocks may have derived from $\sim 8 \%$ partial melting of a theoretical slightly enriched source in the spinel-facies. P-MORBs and alkaline basalts have REE compositions that can be accounted for by mixing of different proportions of melts generated in the garnet- and spinel-facies from an enriched mantle source metasomatized by OIB-type (plume-type) components.

(4) The coexistence of chemically different rock types from Middle Jurassic to Early Cretaceous times suggests that they were formed in a mid-ocean ridge setting from partial melting of a highly heterogeneous mantle characterized by the extensive occurrence of OIB-metasomatized portions, which were likely inherited from a Triassic mantle plume activity associated with the continental rift and opening of the Neotethys branch.

Acknowledgements. The Italian Ministry of Education, University and Research (MIUR) is acknowledged for the financial support (Prin 2010-2011). This research has also been funded by the Ferrara University (FIR-2016 Project). Many thanks go to Mirella Bonora (Ferrara University) for her support with analytical techniques. Radiolarian micrographs were taken with a Philips XL20 of the Ivalsa Institute (CNR) by Simona Lazzeri and with a Zeiss EVO MA15 of the MEMA (University of Florence) by Maurizio Ulivi. Special thanks go to Špela Goričan and anonymous reviewer for their constructive reviews of this paper.

\section{Supplementary material}

To view supplementary material for this article, please visit https://doi.org/10.1017/S0016756817000401.

\section{References}

Akal, C., Candan, O., Koralay, E., Oberhänsli, R., Chen, F. \& Prelevic, D. 2012. Early Triassic potassic volcanism in the Afyon Zone of the Anatolides/Turkey: implications for the rifting of the Neo-Tethys. International Journal of Earth Sciences 101, 177-94.

AitA, Y. \& OKADA, H. 1986. Radiolarians and calcareous nannofossils from the Uppermost JurassicLower Cretaceous strata of Japan and Tethyan Regions. Micropaleontology 32, 97-128.

Akbayram, K., OKay, A. I. \& SatiR, M. 2012. Early Cretaceous closure of the Intra-Pontide Ocean in western Pontides (northwestern Turkey). Journal of Geodynamics 65, 38-55.

Aliev, K. 1967. New radiolarian species of the Valanginian and Albian Stages of northeastern Azerbaidzhan. In Cretaceous Deposits of the Eastern Caucasus and Adjacent Areas (Biostratigraphy and Paleogeography), pp. 23-30. Academy of Sciences USSR, Ministry of Oil Industry USSR, Institute of Geology and of the Processing of Fuel Minerals, Laboratory of the Biostratigraphy of Oil- and Gas-bearing Areas.

Allahyari, K., Saccani, E., Pourmoafi, M., Beccaluva, L. \& MAsoudi, F. 2010. Petrology of mantle peridotites and intrusive mafic rocks from the Kermanshah ophiolitic complex (Zagros belt, Iran): implications for the geodynamic evolution of the Neo-Tethyan oceanic branch between Arabia and Iran. Ofioliti 35, 71-90.

Allègre, C. J. \& Minster, J. F. 1978. Quantitative models of trace element behaviour in magmatic processes. Earth and Planetary Science Letters 38, 1-25.

Altiner, D., Koçyiğit, A., Farinacci, A., Nicosia, U. \& ConTI, M. A. 1991. Jurassic-Lower Cretaceous stratigraphy and paleogeographic evolution of the southern part of north-western Anatolia (Turkey). Geologica Romana 27, 13-80.

Bailey, E. B. \& McCallien, W. J. 1953. Serpentinite lavas, the Ankara Mélange, and the Anatolian Thrust. Transactions of the Royal Society of Edinburgh 57, 403-42.

BAK, M. 1996. Cretaceous Radiolaria from Niedzica Succession of the Pieniny Klippen Belt in Polish Carpathians. Acta Palaeontologica Polonica 41, 91-110.

BAK, M. 1999. Cretaceous radiolarian zonation in the Polish part of the Pieniny Klippen Belt (Western Carpathians). Geologica Carpathica 50, 21-31.

Bandini, A. N., Baumgartner, P. O., Flores, K., DumitricA, P. \& JACKeTt, S. J. 2011. Early Jurassic to early Late Cretaceous radiolarians from the Santa Rosa accretionary complex (northwestern Costa Rica). Ofioliti 31, 1-35.

Baumgartner, P. O., Bartolini, A. C., Carter, E. S., Conti, M., Cortese, G., Danelian, T., De Wever, P., Dumitrica, P., Dumitrica-Jud, R., Goričan, Š., Guex, J., Hull, D. M., Kito, N., Marcucci, M., Matsuoka, A., Murchey, B., O’Dogherty, L., SaVARY, J., Vishnevskaya, V., WidZ, D. \& YaO, A. 1995a. Middle Jurassic to Early Cretaceous radiolarian biochronology of Tethys based on Unitary Associations. In Middle Jurassic to Lower Cretaceous Radiolaria of Tethys: Occurrences, Systematics, Biochronology (eds P. O. Baumgartner, L. O'Dogherty, Š. Goričan, E. Urquhart, A. Pillevuit \& P. De Wever), pp. 1013-48. Mémoires de Géologie, Lausanne, no. 23.

Baumgartner, P. O., Buørklund, K. R., Caulet, J. P., De Wever, P., KellogG, D., Labracherie, M., Nakaseko, K., Nishimura, A., SchaAf, A., Schimdt-Effing, R. \& YAO, A. 1981. Eurorad II, 1980. Second European 
meeting of radiolarian paleontologists: current research on Cenozoic and Mesozoic radiolarians. Eclogae Geologicae Helvetiae 74, 1027-61.

Baumgartner, P. O., O'Dogherty, L., Goričan, Š., Dumitrica-Jud, R., Dumitrica, P., Pillevuit, A., Urquhart, E., Matsuoka, A., Danelian, T., Bartolini, A. C., Carter, E. S., De Wever, P., Kito, N., Marcucci, M. \& Steiger, T. A. $1995 b$. Radiolarian catalogue and systematics of Middle Jurassic to Early Cretaceous Tethyan genera and species. In Middle Jurassic to Lower Cretaceous Radiolaria of Tethys: Occurrences, Systematics, Biochronology (eds P. O. Baumgartner, L. O’Dogherty, Š. Goričan, E. Urquhart, A. Pillevuit \& P. De Wever), pp. 37-685. Mémoires de Géologie, Lausanne, no. 23.

Beker, J. A., Menzies, M. A., Thirlwall, M. F. \& MacPherson, C. G. 1997. Petrogenesis of Quaternary intraplate volcanism, Sana'a, Yemen: implications for plume-lithosphere interaction and polybaric melt hybridization. Journal of Petrology 38, 1359-90.

Berber, F., Göncüoglu, M. C. \& Sayit, K. 2014. Geochemistry and tectonic significance of the Kösedag metavolcanic rocks from the Sakarya Zone, Northern Turkey. Bulletin. In Proceedings of the 20th CBGA Congress 24-26 Sept 2014, Tirana (eds A. Begiraj, C. Ionescu, G. Christofides, A. Uta, E. Beqiraj Goga \& S. Marku). Shkencave Gjeologjike Special Issue 2, $161-3$.

Boccaletti, M., Bortolotti, V. \& Sagri, M. 1966. Ricerche sulle ofioliti delle catene alpine: I. Osservazioni sull'Ankara Mélange nella zona di Ankara. Bollettino della Società Geologica Italiana $\mathbf{8 5}, 485-508$.

BortolotTI, V. \& Principi, G. 2005. Tethyan ophiolites and Pangea break-up. The Island Arc 14, 442-70.

Bortolotti, V., Chiari, M., GöncüOglu, M. C., Marcucci, M., Principi, G., Tekin, U. K., Saccani, E. \& TASsinari, R. 2013a. Age and geochemistry of basalt-chert associations in the ophiolitic complexes of the Izmir-Ankara Mélange East of Ankara, Turkey: preliminary data. Ofioliti 38, 157-73.

Bortolotti, V., Chiari, M., Marroni, M., Pandolfi, L., Principi, G. \& Saccani, E. 2013b. Geodynamic evolution of the ophiolites from Albania and Greece (Dinaric-Hellenic belt): one, two or more oceanic basins? International Journal of Earth Sciences 102, 783-811.

Bortolotti, V. \& Sagri, M. 1968. Ricerche sulle ofioliti delle catene alpine. 4 - Osservazioni sull'età e la giacitura delle ofioliti tra Smirne ed Erzurum (Turchia). Bollettino della Società Geologica Italiana 87, 661-6.

Bragin, N. Yu. \& Tekin, U. K. 1996. Age of radiolarianchert blocks from the Senonian ophiolitic mélange (Ankara, Turkey). The Island Arc 5, 114-22.

Brunelli, D., Seyler, M., Cipriani, A., Ottolini, L. \& BonATTI, E. 2006. Discontinuous melt extraction and weak refertilization of mantle peridotites at the Vema Lithospheric Section (Mid-Atlantic Ridge). Journal of Petrology 47, 745-71.

Catanzariti, R., Ellero, A., Göncüoglu, M. C., Marroni, M., Ottria, G. \& Pandolfi, L. 2013. The Taraklı Flysch in the Boyali area (Sakarya Terrane, northern Turkey): implications for the tectonic history of the IntraPontide suture zone. Comptes Rendus Geoscience, 345, 454-61.

Cater, J. M. L., Hanna, S. S., Ries, A. C. \& Turner, P. 1991. Tertiary evolution of the Sivas Basin, central Turkey. Tectonophysics 195, 29-46.
Cemen, I., GöncüoĞlu, M. C., Erler, A., Kozlu, H. \& PERINÇEK, D. 1993. Indentation tectonics and associated lateral extrusion in East, Southeast and Central Anatolia. Geological Society of America, Programs and Abstracts, A116-7.

Chiari, M., Bortolotti, V., Marcucci, M., Photiades, A., PRINCIPI, G. \& SACCANI, E. 2012. Radiolarian biostratigraphy and geochemistry of the Koziakas Massif ophiolites (Greece). Bulletin de la Societé géologique de France 183, 289-309.

Chiari, M., Cobianchi, M. \& Picotti, V. 2007. Integrated stratigraphy (radiolarians and calcareous nannofossils) of the Middle to Upper Jurassic Alpine radiolarites (Lombard Basin, Italy): constraints to their genetic interpretation. Palaeogeography, Palaeoclimatology, $\mathrm{Pa}$ laeoecology 249, 233-70.

Chiari, M., Marcucci, M. \& Prela, M. 2004. Radiolarian assemblages from the Jurassic cherts of Albania: new data. Ofioliti 29, 95-105.

Danelian, T. 2008. Diversity and biotic changes of Archaeodictyomitrid Radiolaria from the Aptian/Albian transition (OAE1b) of southern Albania. Micropaleontology 54, 3-13.

Danelian, T., Tsikos, H., Gardin, S., Baudin, F., Bellier, J. P. \& Emmanuel, L. 2004. Global and regional palaeoceanographic changes as recorded in the MidCretaceous (Aptian-Albian) sequence of the Ionian zone (NW Greece). Journal of the Geological Society, London 161, 703-9.

Dercourt, J., Zonenshain, L. P., Ricou, L. E., Kazminm, V. G., Le Pichon, X., KnipPer, A. L., Grandjacquet, C., Sbortshikovm, I. M., Geyssant, J., Lepvrier, C., Pechersky, D. H., Boulin, J., Sibuet, J. C., Savostin, L. A., Sorokhtin, O., Westphal, M., Bazhenov, M. L., Lauer, J. P. \& Biju-Duval, M. B. 1986. Geological evolution of the Tethys belt from the Atlantic to the Pamirs since the Lias. In Evolution of the Tethys(eds J. Aubouin, X. Le Pichon \& A. S. Monin). Tectonophysics 123, 241-315.

De Wever, P. 1982. Radiolaires du Trias et du Lias de la Téthys (systématique, stratigraphie). Societé Géologique du Nord 7, 1-600.

Dilek, Y., Thy, P., Hacker, B. \& Grundvig, S. 1999. Structure and petrology of Tauride ophiolites and mafic dike intrusions (Turkey): implications for the Neo-Tethyan Ocean. Geological Society of America Bulletin 111, 1192-216.

DumitricA, P. 1970. Cryptocephalic and cryptothoracic Nassellaria in some Mesozoic deposits of Romania. Revue Roumaine de Géologie, Géophysique et Géographie (série Géologie) 14, 45-124.

Dumitrica, P. \& Dumitrica-Jud, R. 1995. Aurisaturnalis carinatus (Foreman), an example of phyletic gradualism among Saturnalid-type radiolarians. Revue de Micropaléontologie 38, 195-216.

Dumitrica, P., Immenhauser, A. \& Dumitrica-Jud, R. 1997. Mesozoic radiolarian biostratigraphy from Masirah Ophiolite, Sultanate of Oman Part I: Middle Triassic, uppermost Jurassic and Lower Cretaceous Spumellarians and multisegmented Nassellarians. Bulletin of the National Museum of Natural Sciences, Taiwan 9, 1-106.

DumitricA, P. \& ZüGEL, P. 2008. Early Tithonian Saturnalidae (Radiolaria) from the Solnhofen area (Southern Franconian Alb, southern Germany). Paläontologische Zeitschrift 82, 55-84.

Ellero, A., Ottria, G., Marroni, M., Pandolfi, L. \& GöncÜOĞLU, M. C. 2015a. Analysis of the North 
Anatolian Shear Zone in Central Pontides (northern Turkey): insight for geometries and kinematics of deformation structures in a transpressional zone. Journal of Structural Geology 72, 124-41.

Ellero, A., Ottria, G., Sayit, K., Catanzariti, R., Frassi, C., GöncüoĞLU, M. C., Marroni, M. \& Pandolfi, L. 2015b. Geological and geochemical evidence for a Late Cretaceous continental arc in the central Pontides, northern Turkey. Ofioliti 40, 73-90.

Elmas, A. \& YıĞıtbaş, E. 2001. Ophiolite emplacement by strike-slip tectonics between the Pontide Zone and the Sakarya Zone in northwestern Anatolia, Turkey. International Journal of Earth Sciences 90, 257-69.

Elmas, A. \& YiĞITbaş, E. 2005. Comment on "Tectonic evolution of the Intra-Pontide suture zone in the Armutlu Peninsula, NW Turkey" by Robertson \& Ustaömer. Tectonophysics 405, 213-21.

Erdogan, B., Akay, C \& UGur, M. S. 1996. Geology of the Yozgat region and evolution of the collisional Çankırı basin. International Geological Revue 38, 788-806.

Filippov, A. N. \& Kemkin, I. V. 2005. First finds of Middle Jurassic and Early Cretaceous (Valanginian) radiolarian assemblages in the Western Sikhote-Alin: their paleogeographic and tectonic significance. Doklady Earth Sciences 405, 1141-4.

Floyd, P. A., Göncüoglu, M. C., Winchester, J. A. \& YALINIZ, M. K. 2000. Geochemical character and tectonic environment of Neotethyan ophiolitic fragments and metabasites in the Central Anatolian Crystalline Complex, Turkey. In Tectonics and Magmatism in Turkey and the Surrounding Area (eds E. Bozkurt, J. Winchester \& J. A. Piper.), pp. 183-202. Geological Society of London, Special Publication no. 173.

GöKten, E. \& Floyd, P. A. 2007. Stratigraphy and geochemistry of pillow basalts within the ophiolitic mélange of the Izmir-Ankara-Erzincan suture zone: implications for the geotectonic character of the northern branch of Neotethys. International Journal of Earth Sciences (Geologische Rundschau) 96, 725-41.

GöNCÜOGLU, M.C. 1992. Structural and stratigraphical framework of the Central Anatolian Tertiary basins. In Introduction to the Early Paleogene of the HaymanaPolatlı Basin (eds E. Sirel \& E. Yazgan), pp. 1-11. Field Trip Guidebook. Ankara: General Direction of Mineral Resources Exploration.

GöncüOGLU, M. C. 2010. Introduction to the Geology of Turkey: Geodynamic Evolution of the Pre-Alpine and Alpine Terranes. General Directorate of Mineral Resources Exploration Monography Series 5, 66 pp.

GöNcüOGLU, M. C. 2011. Geology of the KütahyaBolkardağ Belt. Mineral Resources Exploration Bulletin 142, 223-77.

GöncüOGlu, M. C., Dirik, K. \& Kozlu, H. 1997. General characteristics of pre-Alpine and Alpine Terranes in Turkey: explanatory notes to the terrane map of Turkey. Annales Géologiques des Pays Helléniques 37, 515-36.

Göncüoglu, M. C., Gürsu, S., Tekin, U. K. \& KöKsal, S. 2008. New data on the evolution of the Neotethyan oceanic branches in Turkey: late Jurassic ridge spreading in the Intra-Pontide branch. Ofioliti 33, 153-64.

Göncüoglu, M. C., Marroni, M., Pandolfi, L., Ellero, A., Ottria, G., Catanzariti, R., Tekin, U. K. \& Sayit, K. 2014. The Arkot Dağ Mélange in Araç area, central Turkey: evidence of its origin within the geodynamic evolution of the Intra-Pontide suture zone. Journal of Asian Earth Sciences 85, 117-39.

Göncüoglu, M. C., Marroni, M., SAYit, K., Tekin, U. K., Pandolfi, L. \& Ellero, A. 2012. The Ayli Dağ ophi- olite sequence (Central-Northern Turkey): a fragment of Middle Jurassic oceanic lithosphere within the IntraPontide Suture Zone. Ofioliti 37, 77-92.

GöncüOglu, M. C., SaYit, K. \& TeKIn, U. K. 2010. Oceanization of the northern Neotethys: geochemical evidence from ophiolitic mélange basalts within the İzmirAnkara suture belt, NW Turkey. Lithos 116, 175-87.

Göncüoglu, M. C., TeKIN, U. K., SAYIT, K., Bedi, Y. \& UzUNÇIMEN, S. 2015. Opening, evolution and closure of the Neotethyan oceanic branches in Anatolia as inferred by radiolarian research. Radiolaria 35, 88-90.

Göncüoglu, M. C., Tekin, U. K. \& Turhan, N. 2001. Geological meaning of the Late Carnian basalts blocks with radiolarites within the Late Cretaceous Central Sakarya ophiolitic complex (NW Anatolia). Jeo 2000 Proceedings CD-54-6: 6.

GöncüOglu, M. C., Turhan, N., Sentürk, K., Özcan, A. \& Uysal, S. 2000. A geotraverse across NW Turkey: tectonic units of the Central Sakarya region and their tectonic evolution. In Tectonics and Magmatism in Turkey and the Surrounding Area (eds E. Bozkurt, J Winchester \& J.A. Piper), pp. 139-61. Geological Society of London, Special Publication no. 173.

GöncüOglu, M. C., Turhan, N. \& Tekin, U. K. 2003. Evidence for the Triassic rifting and opening of the Neotethyan Izmir-Ankara Ocean, northern edge of the Tauride-Anatolide platform, Turkey. Bollettino della Società Geologica Italiana, Special Volume 2, 203-12.

Göncüoglu, M. C., Yaliniz, K. \& TeKIn, U. K. 2006. Geochemistry, tectono-magmatic discrimination and radiolarian ages of basic extrusives within the IzmirAnkara Suture Belt (NW Turkey): time constraints for the Neotethyan evolution. Ofioliti 31, 25-38.

GoriČAN, Š. 1994. Jurassic and Cretaceous radiolarian biostratigraphy and sedimentary evolution of the Budva Zone (Dinarides, Montenegro). Mémoires de Géologie, Lausanne 18, 1-120.

Goričan, Š., Pavšıč, J. \& Rožıč, B. 2012. Bajocian to Tithonian age of radiolarian cherts in the Tolmin basin (NW Slovenia). Bulletin de la Société géologique de France 183, 369-82.

Gülyüz, E., Kaymakci, N., MeiJers, M. J. M., van HinsBergen, D. J. J., Lefebvre, C., Vissers, R. L. M., BART, W. H., Hendriks, B. W. H. \& Peynircioglu, A. A. 2013. Late Eocene evolution of the Çiçekdağ (central Turkey): syn-sedimentary compression during microcontinent-continent collision in central Anatolia. Tectonophysics. 602, 286-99.

Hanan, B. B., Blichert-Toft, J., Kingsley, R. \& Schilling, J. G. 2000. Depleted Iceland mantle plume geochemical signature: artifact of multicomponent mixing? Geochemistry, Geophysics, Geosystems 1, 1-19.

Hubert-Ferrari, A., Armijo, R., King, G., Meyer, B. \& BARKA, A. 2002. Morphology, displacement, and slip rates along the North Anatolian Fault, Turkey. Journal of Geophysical Research 107, ETG 9-1-ETG 9-33.

Irving, A. J. \& FreY, F. A. 1984. Trace element abundances in megacrysts and their host basalts: constraints on partition coefficients and megacryst genesis. Geochimica et Cosmochimica Acta 48, 1201-21.

JACKsON, M. L. 1958. Soil Chemical Analysis. Englewood Cliffs, NJ: Prentice-Hall, 498 pp.

Kawabata, K. 1988. New species of Latest Jurassic and Earliest Cretaceous radiolarians from the Sorachi group in Hokkaido, Japan. Bulletin of the Osaka Museum of Natural History 43, 1-13.

Kaymakci, N., Duermeijer, C. E., Langereis, C., White, S. H. \& VAN DIJK, P. M. 2003. Palaeomagnetic 
evolution of the Çankırı Basin (Central Anatolia, Turkey: implication for oroclinal bending due to indentation. Geological Magazine 140, 343-55.

Kocyigit, A., Türkmenoglu, A., Beyhan, A., Kaymakçı, N. \& AKyol, E. 1995. Post-collisional tectonics of Eskisehir-Ankara-Çankırı segment of Izmir-AnkaraErzincan Suture Zone. Turkish Association of Petroleum Geologists Bulletin 6, 69-87.

KöKsal, S. \& GöNCüoglu, C. M. 2008. Sr and Nd isotopic characteristics of some S-, I- and A-type granitoids from Central Anatolia. Turkish Journal of Earth Sciences 17, 111-27.

Kozur, H. 1985. The radiolarian genus Eoxitus n. gen from the Unuma echinatus zone (Bajocian) of Northern Hungary. Proceed Koninklijke Nederoands Academie Van Wetenscappen, 88, 211-20.

Lachance, G. R. \& Trail, R. J. 1966. Practical solution to the matrix problem in X-ray analysis. Canadian Spectroscopy 11, 43-48.

Liu, C.-Z., Snow, J. E., Hellebrand, E., Brü̈mann, G. E., von DER HandT, A. B. \& Hofmann, A. W. 2008. Ancient, highly heterogeneous mantle beneath Gakkel ridge. Arctic Ocean. Nature 452 , 311-6.

McKenzie, D. \& O’Nions, R. K. 1991. Partial melt distributions from inversion of rare Earth element concentrations. Journal of Petrology 32, 1021-91.

Moix, P., Beccaletto, L., Kozur, H. W., Hochard, C., Rosselet, F. \& Stampfli, G. M. 2008. A new classification of the Turkish terranes and sutures and its implication for the paleotectonic history of the region. Tectonophysics 451, 7-39.

MoIx, P. \& GoriČAN, Š. 2013. Jurassic and Cretaceous radiolarian assemblages from the Bornova mélange in northern Karaburun Peninsula (western Turkey) and its connection to the İzmir-Ankara mélanges. Geodinamica Acta 26, 56-67.

Montanini, A., Tribuzio, R. \& Vernia, L. 2008. Petrogenesis of basalts and gabbros from an ancient continentocean transition (External Liguride ophiolites, Northern Italy). Lithos 101, 453-79.

O'DogherTy, L. 1994. Biochronology and paleontology of Mid-Cretaceous radiolarians from Northern Apennines (Italy) and Betic Cordillera (Spain). Mémoires de Géologie, Lausanne 21, 1-415.

O'Dogherty, L., Carter, E. S., Dumitrica, P., Goričan, Š., De Wever, P., Bandini, A. N., Baumgartner, P. O. \& Matsuoka, A. 2009. Catalogue of Mesozoic radiolarian genera. Part 2: Jurassic-Cretaceous. Geodiversitas 31, 271-356.

OKaY, A. I. \& GöncüOglu, M. C. 2004. The Karakaya Complex: a review of data and concepts. Turkish Journal of Earth Sciences 13, 75-95.

OKAY, A. I. \& TüYsüz, O. 1999. Tethyan sutures of northern Turkey. In The Mediterranean Basin: Tertiary Extension within the Alpine Orogen (eds B. Durand, L. Jolivet, F. Horváth \& M. Séranne), pp. 475-515. Geological Society of London, Special Publication no. 156.

ParlaK, O., ÇolakoĞlu, A., Dönmez, C., SAYAK, H., Yildirim, N., Türkel, A. \& OdABAŞI, İ. 2013. Geochemistry and tectonic significance of ophiolites along the İzmir-Ankara-Erzincan Suture Zone in northeastern Anatolia. In Geological Development of Anatolia and the Easternmost Mediterranean (eds A. H. F. Robertson, O. Parlak \& U. Unlugenc), pp. 75-105. Geological Society of London, Special Publication no. 372.
Pearce, J. A. 1982. Trace element characteristics of lavas from destructive plate boundaries. In Andesites (ed. R. S. Thorpe), pp. 525-48. New York: John Wiley \& Sons.

Pearce, J. A. \& Norry, M. J. 1979. Petrogenetic implications of $\mathrm{Ti}, \mathrm{Zr}, \mathrm{Y}$, and $\mathrm{Nb}$ variations in volcanic rocks. Contributions to Mineralogy and Petrology 6, 33-47.

Pessagno, E. A. \& Newport, L. A. 1972. A technique for extracting Radiolaria from radiolarian chert. Micropaleontology 18, 231-4.

Robertson, A. H. F., Dixon, J. E., Brown, S., Collins, A., Morris, A., Pickett, E. A., Sharp, I. \& Ustaömer, T. 1996. Alternative tectonic models for the Late Palaeozoic-Early Tertiary development of Tethys in the Eastern Mediterranean region. In Palaeomagnetism and Tectonics of the Mediterranean Region (eds A. Morris \& D. H. Tarling), pp. 239-63. Geological Society of London, Special Publication no. 105.

Robertson, A., Parlak, O., Ustaömer, T., Tasli, K., İnan, N., Dumitrica, P. \& KaraoĞLan, F. 2013. Subduction, ophiolite genesis and collision history of Tethys adjacent to the Eurasian continental margin: new evidence from the Eastern Pontides, Turkey. Geodinamica Acta 26, 230-93.

Robertson, A. H. F. \& Ustä̈mer, T. 2004. Tectonic evolution of the Intra-Pontide suture zone in the Armutlu Peninsula, NW Turkey. Tectonophysics 381, 175-209.

Robin, C., Goričan, Š., Guillocheau, F., Razin, P., Dromart, G. \& Mosaffa, H. 2010. Mesozoic deepwater carbonate deposits from the southern Tethyan passive margin in Iran (Pichakun nappes, Neyriz area): biostratigraphy, facies sedimentology and sequence stratigraphy. In Tectonic and Stratigraphic Evolution of Zagros and Makran during the Mesozoic-Cenozoic (eds P. Leturmy \& C. Robin), pp. 179-210. Geological Society of London, Special Publication no. 330.

RojAY, B. 2013. Tectonic evolution of the Cretaceous Ankara Ophiolitic Mélange during the Late Cretaceous to preMiocene interval in Central Anatolia, Turkey. Journal of Geodynamics 65, 66-81.

RojaY, B., Yaliniz, M. K. \& Altiner, D. 2001. Tectonic implications of some Cretaceous pillow basalts from the North Anatolian Ophiolitic Mélange (Central AnatoliaTurkey) to the evolution of Neotethys. Turkish Journal of Earth Sciences 10, 93-102.

SACCANI, E. 2015. A new method of discriminating different types of post-Archean ophiolitic basalts and their tectonic significance using $\mathrm{Th}-\mathrm{Nb}$ and $\mathrm{Ce}-\mathrm{Dy}-\mathrm{Yb}$ systematics. Geoscience Frontiers 6, 481-501.

Saccani, E., Allahyari, K., Beccaluva, L. \& Bianchini, G. 2013a. Geochemistry and petrology of the Kermanshah ophiolites (Iran): implication for the interaction between passive rifting, oceanic accretion, and plumecomponents in the Southern Neo-Tethys Ocean. Gondwana Research 24, 392-411.

Saccani, E., Azimzadeh, Z., Dilek, Y. \& Jahangiri, A. $2013 b$. Geochronology and petrology of the Early Carboniferous Misho Mafic Complex (NW Iran), and implications for the melt evolution of Paleo-Tethyan rifting in Western Cimmeria. Lithos 162-163, 264-78.

Saccani, E., Beccaluva, L., Photiades, A. \& Zeda, O. 2011. Petrogenesis and tectono-magmatic significance of basalts and mantle peridotites from the AlbanianGreek ophiolites and sub-ophiolitic mélanges. New constraints for the Triassic-Jurassic evolution of the Neo-Tethys in the Dinaride sector. Lithos 124, 227-42.

Saccani, E., Delavari, M., Beccaluva, L. \& Amini, S. A. 2010. Petrological and geochemical constraints on the origin of the Nehbandan ophiolitic complex (eastern 
Iran): implication for the evolution of the Sistan Ocean. Lithos 117, 209-28.

Saccani, E. \& Photiades, A. 2005. Petrogenesis and tectono-magmatic significance of volcanic and subvolcanic rocks in the Albanide-Hellenide ophiolitic mélanges. The Island Arc 14, 494-516.

Saccani, E., Photiades, A., Santato, A. \& Zeda, O. 2008a. New evidence for supra-subduction zone ophiolites in the Vardar Zone from the Vermion Massif (northern Greece): implication for the tectonomagmatic evolution of the Vardar oceanic basin. Ofioliti 33, 65-85.

SacCANI, E. \& Principi, G. 2016. Petrological and tectonomagmatic significance of ophiolitic basalts from the Elba Island within the Alpine Corsica-Northern Apennine system. Mineralogy and Petrology 110, 713-30.

Saccani, E., Principi, G., Garfagnoli, F. \& Menna, F. $2008 b$. Corsica ophiolites: geochemistry and petrogenesis of basaltic and metabasaltic rocks. Ofioliti 33, 187207.

SaYit, K., Tekin, U. K. \& GöncüOglu, M. C. 2011. EarlyMiddle Carnian radiolarian cherts within the Eymir Unit, Central Turkey: constraints for the age of the Palaeotethyan Karakaya Complex. Journal of Asian Earth Sciences 42, 398-407.

Schmid, S. M., Bernoulli, D., Fügenschuh, B., Matenco, L., Schefer, S., Schuster, R., Tischler, M. \& UstaszewsKI, K. 2008. The Alpine-CarphatianDinaridic orogenic system: correlation and evolution of tectonic units. Swiss Journal of Geosciences 101, 139-83.

SengöR, A. M. C. \& YiLmaz, Y. 1981. Tethyan evolution of Turkey: a plate tectonic approach. Tectonophysics $\mathbf{7 5}$, 181-241.

Smuc, A. \& Goričan, Š. 2005. Jurassic sedimentary evolution of a carbonate platform into a deep-water basin, Mt. Mangart (Slovenian-Italian Border). Rivista Italiana di Paleontologia e Stratigrafia 111, 45-70.

Soycan, H., ERdoĞan, K. \& KonAK, N. 2015. AalenianEarly Bathonian (Middle Jurassic) radiolarian assemblages in the Tavas nappe within Lycian nappes in the western Taurides (SW Turkey): the first dating of carbonate platform drowning. Journal of Asian Earth Sciences, 104, 3-21.

Stampfli, G. M. \& Borel, G. D. 2002. A plate tectonic model for the Palaeozoic and Mesozoic constrained by dynamic plate boundaries and restored synthetic oceanic isochrones. Earth and Planetary Science Letters 169, 17-33.

Sun, S. S. \& McDonough, W. F. 1989. Chemical and isotopic-systematics of oceanic basalts: implications for mantle composition and processes. In Magmatism in the Ocean Basins (eds A. D. Saunders \& M. J. Norry), pp. 313-45. Geological Society of London, Special Publication no. 42

TeKIN, U. K. 1999. Biostratigraphy and systematics of Late Middle to Late Triassic radiolarians from the Taurus Mountains and Ankara region, Turkey. Geologische und Paläontologische Mitteilungen Innsbruck, Sonderband 5, 1-296.

Tekin, U. K. \& Göncüoglu, M. C. 2007. Discovery of the oldest (Upper Ladinian to Middle Carnian) radiolarian assemblages from the Bornova Flysch Zone in western Turkey: implications for the evolution of the Neotethyan Izmir-Ankara Ocean. Ofioliti 32, 131-50.

Tekin, U. K. \& GöncüOGlu, M. C. 2009. Late Middle Jurassic (late Bathonian-early Callovian) radiolarian cherts from the Neotethyan Bornova Flysch Zone, Spil
Mountains, Western Turkey. Stratigraphical and Geological Correlation 17, 298-308.

Tekin, U. K., GöncüoĞlu, M. C., PAndolfi, L. \& MARroni, M. 2012. Middle-Late Triassic radiolarian cherts from the Arkotdağ mélange in northern Turkey: implications for the life span of the northern Neotethyan branch Geodinamica Acta 25, 305-19.

Tekin, U. K., Göncüoglu, M. C. \& Turhan, N. 2002. First evidence of Late Carnian radiolarian fauna from the Izmir-Ankara Suture Complex, Central Sakarya, Turkey: implications for the opening age of the IzmirAnkara branch of Neotethys. Geobios 35, 127-35.

Tekin, U. K., GöncüOGlu, M. C \& Uzuncimen, S. 2012. Radiolarian assemblages of Middle and Late Jurassic to early Late Cretaceous (Cenomanian) ages from an olistolith record pelagic deposition within the Bornova Flysch Zone in western Turkey. Bulletin de la Societé géologique de France 183, 307-18.

Thirlwall, M., Upton, B. G. J. \& Jenkins, C. 1994. Interaction between continental lithosphere and the Iceland plume-Sr-Nd-Pb isotope geochemistry of Tertiary basalts, NE Greenland. Journal of Petrology 35, 83979.

Topuz, G., Çelik, Ö. F., Şengör, A. M. C., Altintaş, İ. E., ZaCK, T., Rolland, Y. \& Barth, M. 2013a. Jurassic ophiolite formation and emplacement as backstop to a subduction-accretion complex in northeast Turkey, the Refahiye ophiolite, and relation to the Balkans ophiolites. American Journal of Science 313, 1054-87.

Topuz, G., Göçmengil, G., Rolland, Y., ÇeliK, Ö. F., ZACK, T. \& SchmitT, A. K. 2013b. Jurassic accretionary complex and ophiolite from northeast Turkey: no evidence for the Cimmerian continental ribbon. Geo$\log y$ 45, 255-8.

WARREN, J. M. 2016. Global variations in abyssal peridotite compositions. Lithos 248-251, 193-219.

Warren, J. M., Shimizu, N., SAKaguchi, C., Dick, H. J. B. \& Nakamura, E. 2009. An assessment of upper mantle heterogeneity based on abyssal peridotite isotopic compositions. Journal of Geophysical Research 114, B12203, doi: 10.1029/2008JB006186.

Wood, D. A. 1980. The application of a Th-Hf-Ta diagram to problems of tectonomagmatic classification and to establishing the nature of crustal contamination of basaltic lavas of the British Tertiary volcanic province. Earth and Planetary Science Letters 50, 11-30.

WORKMAN, R. K. \& HART, S. R. 2005. Major and trace element composition of the DMM. Earth and Planetary Science Letters 2, 53-72.

Yaliniz, M. K., FloYd, P. A. \& Göncüoglu, M. C. 1996. Supra-subduction zone ophiolites of Central Anatolia: geochemical evidence from the Sarıkaraman Ophiolite, Aksaray. Turkish Mineralogical Magazine 60, 697-710.

Yaliniz, K., Göncüoglu, M. C. \& Floyd, P. A. 2000. Geochemistry of volcanic rocks from the Çicekdag Ophiolite, Central Anatolia, Turkey, and their inferred tectonic setting within the northern branch of the Neotethyan ocean. In Tectonics and Magmatism in Turkey and the Surrounding Area (eds E. Bozkurt, J. Winchester \& J. A. Piper), pp. 203-18. Geological Society of London, Special Publication no. 173.

Yaliniz, M. K., Göncüoglu, M. C. \& ÖZKan-Altiner, S. 2000. Formation and emplacement ages of the SSZtype Neotethyan ophiolites in Central Anatolia, Turkey: paleotectonic implications. Geological Journal 35, 53-68. 
Yilmaz, Y., Serdar, H. S., Genc, C., Yigitbas, E., Gurer, F., Elmas, A., Yildirim, M., Bozcu, M. \& Gurpinar, O. 1997. The geology and evolution of the Tokat Massif, south central Pontides, Turkey. International Geological Revue 39, 365-82.
Yolsal-Çevikbilen, S., Biryol, C. B., Beck, S., Zandt, G., Taymaz, T., Adiyaman, H. E. \& Özacar, A. A. 2012. 3-D crustal structure along the North Anatolian Fault Zone in north-central Anatolia revealed by local earthquake tomography. Geophysical Journal International 188, 819-49. 